Check for updates

Cite this: Mater. Adv., 2021, 2, 96

Received 29th September 2020, Accepted 24th November 2020

DOI: $10.1039 / \mathrm{d} 0 \mathrm{ma} 00745 \mathrm{e}$

rsc.li/materials-advances

\section{Recent advances in the field of carbon-based cathode electrocatalysts for $\mathrm{Zn}$-air batteries}

\author{
Xinxin Shu, ${ }^{a}$ Maomao Yang, ${ }^{a}$ Dongxing Tan, ${ }^{a}$ Kwan San Hui, ID ${ }^{b}$ Kwun Nam Hui ${ }^{\mathrm{C}}$ \\ and Jintao Zhang (iD *a
}

\begin{abstract}
Carbon-based catalysts are widely regarded as one of the most promising materials for energy storage and conversion technologies due to their high electrical conductivity as well as tunable microand nanostructures. Developing efficient, low-cost, and durable bifunctional carbon-based electrocatalysts remains challenging. In this review, the recent advances in the field of carbon-based oxygen reduction reaction/oxygen evolution reaction electrocatalysts for $\mathrm{Zn}$-air batteries are briefly reviewed, focusing on the fabrication strategies of carbon-based electrocatalysts. Finally, the present challenges and perspectives in developing advanced bifunctional carbon-based electrocatalysts are outlined.
\end{abstract}

\section{Introduction}

Excessive fossil fuel consumption, escalating energy demands, and environmental concerns have forced us to pursue alternative energy conversion and storage devices. ${ }^{1}$ Rechargeable zinc-air batteries have drawn much attention as promising alternatives for next-generation energy storage systems owing to their high energy density (1086 $\left.\mathrm{W} \mathrm{h} \mathrm{kg}^{-1}\right)$, safety, nontoxicity, and environmental friendliness. ${ }^{2}$ It is an electrochemical energy storage technology with an open-cell structure, which allows continuous, inexhaustible oxygen supply from the atmosphere as the reactant through the redox reaction between the zinc metal and the oxygen to generate electricity. ${ }^{3}$ However, a stable power output and long-term stability have not yet been achieved due to the insufficient activity of air electrodes. The sluggish kinetics of the oxygen reduction reaction (ORR) and oxygen evolution reaction (OER) hinders the overall performances of batteries. Thus, efficient electrocatalysts for the ORR and OER are desired to decrease the reaction energy barrier and increase the activity and stability. ${ }^{4,5}$ Recently developed non-noble-metal-based catalysts, which could exhibit a comparable catalytic activity and a substantially better fuel tolerance than benchmark catalysts $\left(\mathrm{Pt} / \mathrm{RuO}_{2}\right)$, have made great progress in electrochemical energy storage and conversion. ${ }^{6-9}$ Among various catalysts, carbon-based nanomaterials are considered to be the most promising candidates owing to their

\footnotetext{
${ }^{a}$ Key Laboratory for Colloid and Interface Chemistry, Ministry of Education, School of Chemistry and Chemical Engineering, Shandong University, Jinan 250100, China.E-mail: jtzhang@sdu.edu.cn; Fax: +86-531-88361011

${ }^{b}$ School of Engineering, University of East Anglia, NR4 7TJ, UK

${ }^{c}$ Institute of Applied Physics and Materials Engineering, University of Macau, Macau, China
}

superior electrical conductivity, acceptable cost, unique molecular structures with a large surface area, catalytic activities in acidic and alkaline environments, and tunable electronic structures and microstructures. ${ }^{10-13}$ However, pristine carbon is inert and inefficient for practical cathode catalyst application. Thus, much research effort has been devoted to introducing active sites in carbon-based catalysts through doping (e.g., single, dual-, and multi doping), defect engineering, and hybridization. Several reviews have well-summarized the research progress of metal-air batteries or oxygen catalysts, but an up-to-date summary is necessary due to the increase in recent research studies, especially on carbon-based materials. The design strategies of carbon-based electrocatalysts are discussed in this review, emphasizing the latest development of carbon-based bifunctional oxygen catalysts. In addition, the structure-activity relationships are further understood by coupling the experimental results and theoretical calculations. Finally, the current challenges and future prospects of bifunctional carbon-based electrocatalysts are proposed.

\section{Fundamental principles of ORR/OER}

\subsection{Reaction mechanism of ORR}

The oxygen reduction reaction is a vital process for energy conversion and storage techniques with oxygen participation, and its efficiency directly determines the overall performance of devices. In the discharge process of alkaline $\mathrm{Zn}$-air batteries, the oxygen from the atmosphere could diffuse into the porous air cathode through the gas diffusion layer (GDL). The oxygen obtains electrons from the surface of an electrode and is reduced to hydroxide ions at the triple-phase boundary of oxygen (gas), 
electrolytes (liquid), and electrocatalysts (solid). ${ }^{14,15}$ The reaction steps are proposed below.

(i) the 4-electron reaction process:

$$
\mathrm{O}_{2}+\mathrm{H}_{2} \mathrm{O}+4 \mathrm{e}^{-} \rightarrow 4 \mathrm{OH}^{-} \quad\left(E^{\theta}=0.401 \mathrm{~V}\right)
$$

(ii) the 2-plus-2 electron reaction process:

$$
\begin{gathered}
\mathrm{O}_{2}+\mathrm{H}_{2} \mathrm{O}+2 \mathrm{e}^{-} \rightarrow \mathrm{HO}_{2}^{-} \quad\left(E^{\theta}=0.065 \mathrm{~V}\right) \\
\mathrm{HO}_{2}{ }^{-}+\mathrm{H}_{2} \mathrm{O}+2 \mathrm{e}^{-} \rightarrow 3 \mathrm{OH}^{-} \quad\left(E^{\theta}=0.867 \mathrm{~V}\right)
\end{gathered}
$$

or disproportionation

$$
2 \mathrm{HO}_{2}^{-} \rightarrow \mathrm{O}_{2}+2 \mathrm{OH}^{-}
$$

where $E^{\theta}$ is the standard reduction potential versus the standard hydrogen electrode ( $v s$. SHE). Generally speaking, the ORR can proceed through either an effective 4-electron pathway in which $\mathrm{O}_{2}$ is directly reduced to $\mathrm{OH}^{-}$or a 2-plus-2 electron pathway with the generation of $\mathrm{HO}_{2}{ }^{-}$as the byproduct. It can be seen that the ORR pathways may be divided into two scenarios. It is further found that the ORR pathways and mechanisms vary with different catalysts and are associated with the adsorption of $\mathrm{O}_{2}$ at catalytic active sites to form the intermediates. ${ }^{13}$

\subsection{Reaction mechanism of OER}

The oxygen evolution reaction can be regarded as the reverse process of the ORR, in which $\mathrm{OH}^{-}$is oxidized into $\mathrm{O}_{2}$ in an alkaline electrolyte. The OER is also a complex electrochemical reaction involving multistep electron transfer processes. For practical Zn-air battery applications, the polarization of the OER is more severe than that of the ORR, which will result in a low round-trip efficiency. It is further found that the major issue for battery failure would be attributed to the high charging overpotential along with the OER, which can result in the premature decomposition of a catalyst substrate and/or an electrolyte. ${ }^{14}$ The basic reaction step is shown below.

$$
4 \mathrm{OH}^{-} \rightarrow \mathrm{O}_{2}+2 \mathrm{H}_{2} \mathrm{O}+4 \mathrm{e}^{-} \quad\left(E^{\theta}=0.401 \mathrm{~V}\right)
$$

\section{Rational material design strategies}

\subsection{Heteroatom doping}

In principle, large specific surface areas, good conductivity, low cost, abundant porosity structure, high activities, and stabilities should be identified for designing highly efficient carbon-based electrocatalysts. Metal-free carbon materials with heteroatom doping such as $\mathrm{N}, \mathrm{P}, \mathrm{B}, \mathrm{O}, \mathrm{S}$, and $\mathrm{F}$ heteroatoms (Fig. 1), and even the trace transition metal (Co) doping carbon, could maintain a high surface area, excellent activity, and electrical properties; tune the electronic structure and atomic coordination of the carbon matrix; and are commonly regarded as promising, effective alternatives to Pt-based catalysts for electrocatalysis of the ORR in metal-air batteries and fuel cells. In addition, nitrogen doping has a positive effect on the increase of ORR electrocatalytic activity due to the dipole effect, in which the electron density of the carbon atoms adjacent to the nitrogen atom decreases, favoring the dissociative chemisorption

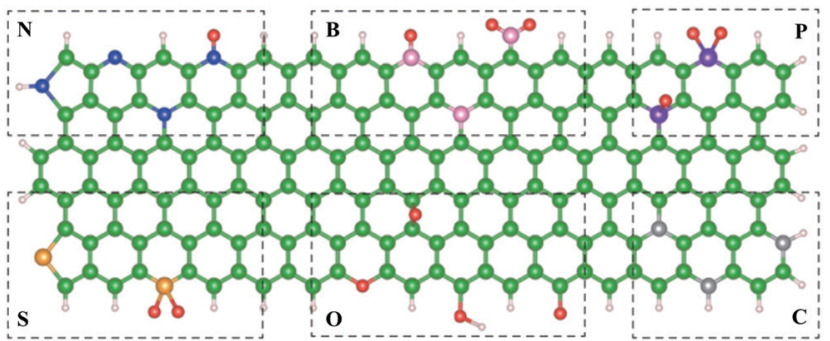

Fig. 1 Schematic summary of heteroatom doping configurations. Reproduced with permission from ref. 17. Copyright 2016, Nature Publishing Group.

of oxygen. ${ }^{5,11,13,16-18}$ To date, carbon-based metal-free catalysts with heteroatom doping have been obtained by changing various heteroatom-enriched dopant precursors or pyrolysis in a desired atmosphere (e.g., $\mathrm{NH}_{3}$ for $\mathrm{N}$ doping, $\mathrm{H}_{2} \mathrm{~S}$ and $\mathrm{SO}_{2}$ for $\mathrm{S}$ doping). ${ }^{19-22}$ However, conventional hydrothermal carbonization or high-temperature pyrolysis remains restricted in terms of two main issues. One issue is the hindered active centers, damaged pore structure, and reduced surface area. The other issue is the difficulty in controlling the location and stability of the active sites. $^{23}$ Generally, the configuration, location, and species of main dopants play a key role in the reaction efficiencies. Studies demonstrated that dual- and multi-doped carbon materials manifest superior electrochemical activity to single doped materials. For example, Zhang et al. developed a large surface area $\left(\sim 1663 \mathrm{~m}^{2} \mathrm{~g}^{-1}\right)$ mesoporous carbon foam that could greatly promote the ORR/OER bifunctional activity by efficient $\mathrm{P}$ doping with N. ${ }^{24}$ Furthermore, density functional theory (DFT) calculations revealed the essential roles of $\mathrm{N}, \mathrm{P}$ codoping, and graphene edge effects. N,P-co-doped highly porous carbon can also be used as a Zn-air battery cathode electrocatalyst with a large power density and a prolonged cycling life. ${ }^{25}$ Thus, doping sites and species of graphene carbon edges are beneficial for enhancing the catalytic activity for the ORR and OER at low overpotentials compared with inner doping. Doping trace transition metals could substantially improve the electrocatalytic activity owing to the modification of the local electronic structure of the carbon surface given the different effects of metals and non-metals on the carbon matrix. ${ }^{18,26}$ Zhang's group developed a hierarchically porous Co, $\mathrm{N}$ co-doped carbon nanoframework (denoted as Co, N-CNF) by a calcination strategy with mesoporous silica protection. ${ }^{23}$ The synergistic effect of $\mathrm{Co}$ and $\mathrm{N}$ could further induce an uneven charge or spin redistribution of the adjacent carbon atoms, facilitating the oxygen adsorption and/or the subsequent $\mathrm{O}-\mathrm{O}$ bond breaking, and thus enhancing the ORR activity. Such a strategy provides a direction for designing highly efficient metal, nitrogen-co-doped carbon electrocatalysts for the ORR and OER.

\subsection{Defect engineering}

Defective carbon-based nanomaterials have attracted much attention recently because they can alter and optimize the adsorption energy of intermediate species, and modulate the electronic structure and surface/interface properties to promote ORR activity. ${ }^{27,28}$ Generally, the classification of defect types is 
complicated, and the commonly accepted, extensively studied ones are divided into intrinsic and non-intrinsic defects. ${ }^{28-30}$ Intrinsic defects refer to atoms at lattice positions that miss due to thermal vibration to form a point defect, containing holes, edges (armchair and zigzag edges), and vacancies, whereas non-intrinsic defects are externally introduced defects usually caused by foreign atoms covalently bonded to carbon atoms in the lattice. ${ }^{31,32}$ Many researchers have demonstrated that the carbon defect is an efficient, crucial strategy to access a highly satisfactory electrocatalytic reactivity for $\mathrm{Zn}$-air batteries. For instance, Qiao's group revealed that the doping content of pyridinic $\mathrm{N}$ can be considerably improved due to the increased edge structure concentration via laser irradiation, which is favorable for the enhancement of the stability and ORR/OER bifunctional catalytic activity (Fig. 2a). ${ }^{30}$ Mu's group reported that the intrinsic pentagon defects in the basal plane can be conducive to the local electronic redistribution and the contraction of the bandgap, making the carbon matrix possess a superior binding affinity for oxygen as well as larger charge densities, facilitating the performance of the ORR (Fig. 2b and c). ${ }^{33}$ Furthermore, Yao's group demonstrated that carbon defects can be controllably derived by removing heteroatoms from graphene, which is of significance for designing efficient electrocatalysts (Fig. 2d). ${ }^{34}$ The defect creation methods discussed above are based on the in situ synthesis, including the high-temperature hydrothermal treatment, pyrolysis, and post-treatment (such as etching). In addition, plasma is an emerging, attractive surface treatment strategy for creating facile, rapid, and controllable defects. It can effectively alter the surface functional groups and expose more surface sites. ${ }^{35-37}$ Thus, the advantages of defect engineering could be summarized as follows: (1) it regulates physical and/or chemical properties. Introducing defects can effectively alter the porosity structure and composition of carbon nanomaterials, and substantially modify the electrical conductivity and conduction band level. (2) It induces catalytic sites. Different types of defective carbons are key motivations for the electrocatalytic

\section{$\mathbf{a}$}
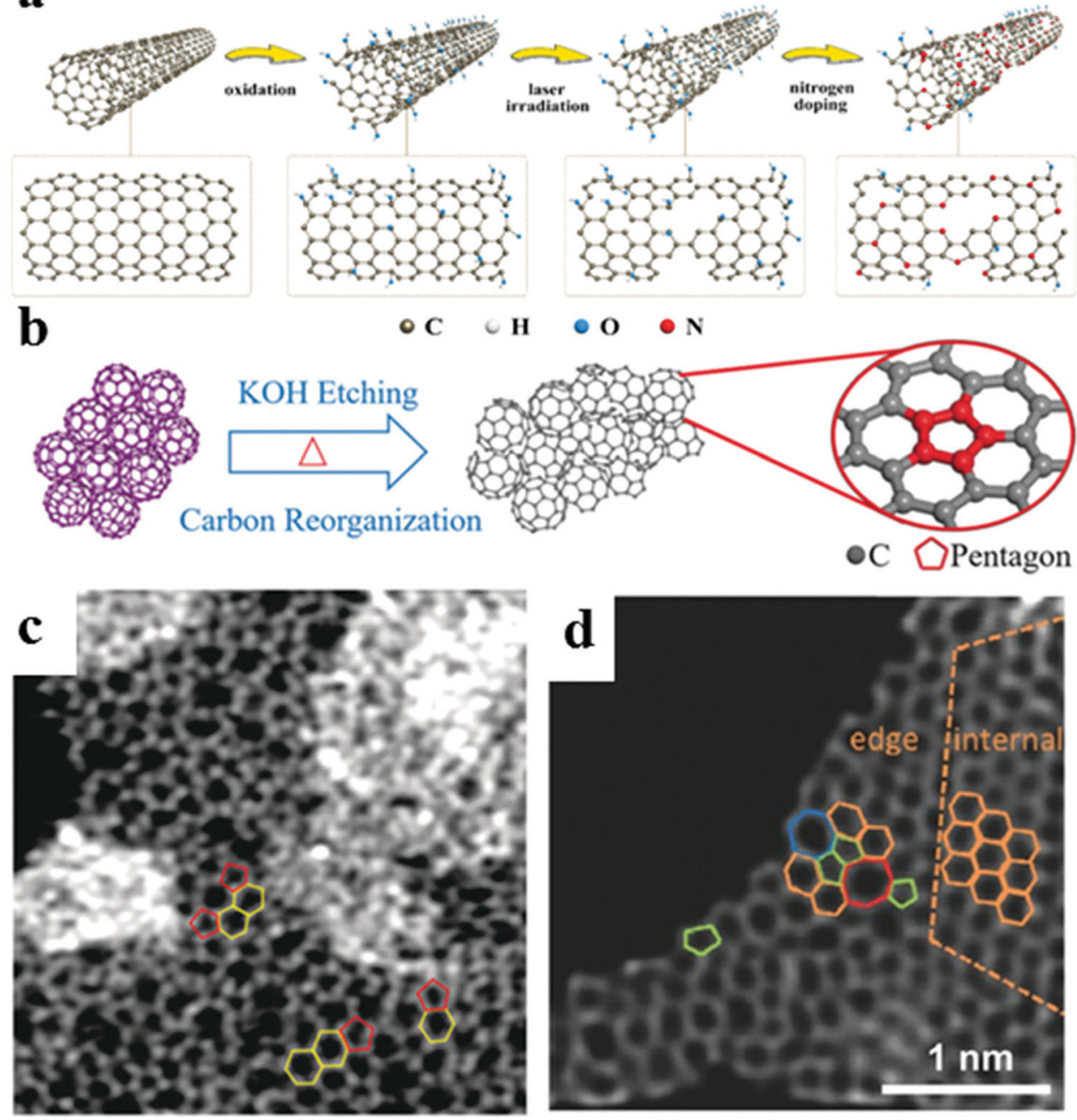

Fig. 2 (a) Schematic illustration of the preparation of laser-induced nitrogen doped carbon nanotube (NL-CNT). Reproduced with permission from ref. 30. Copyright 2019, Wiley-VCH. (b) Schematic of the formation of pentagon defect-rich carbon (PD-C). (c) Cropped image of the ac-STEM image. Reproduced with permission from ref. 33. Copyright 2019, Wiley-VCH. (d) HAADF image of defective graphene. Reproduced with permission from ref. 34. Copyright 2016, Wiley-VCH. 
ORR, OER, and hydrogen evolution reaction (HER). (3) It has outstanding support for coupling with active species. Taking advantage of the synergistic effects of dual components, the optimal electrochemical performance can be realized. However, it is still desirable to develop general, cost-effective synthetic strategies for ingeniously designing the geometric and electronic structures of efficient carbon-based catalysts.

\subsection{Hybridization}

An efficient bifunctional catalyst that is cost effective and earth abundant has long been pursued because it is the core of next generation energy systems. ${ }^{38}$ However, a single active component has difficulty in satisfying the practical application of metal-air batteries and fuel cells because of the premature failure of the active component. Therefore, achieving efficient integration of dual/multi active components into one catalyst is highly desirable to understand the interaction and synergetic effect among the different components. Hybrid materials of transition metals and/or their derivatives with conductive carbon are demonstrated as efficient electrocatalysts to boost the ORR and OER. ${ }^{39,40}$ Typically, carbon-supported catalysts, especially carbon-coated transition metal nanoparticles (NPs) (Fe, Co, and $\mathrm{Ni}$ ) or metal compounds, have been extensively studied and achieved success to some extent. The types of carbon coatings are not limited to graphene layers but also include carbon nanotubes and other derived graphitized carbon layers (such as $\mathrm{C}_{3} \mathrm{~N}_{4}$ ). For example, Wang's group demonstrated that a lower amount of Co species and an extremely low $\mathrm{N}$ doping are likely to enhance the properties with the carbon matrix, achieving a high ORR activity. ${ }^{41}$ In addition, the elaborate design of metal alloys with a wide range of sources that are cost effective, non-toxic, and harmless is indispensable for maximizing the ORR/OER reaction activity of integrated electrocatalysts. Cao's group integrated CoNi bimetallic NPs into $\mathrm{N}$-doped porous carbon to prepare a highly active oxygen catalyst (denoted as CoNi/NG), as shown in Fig. 3a. ${ }^{42}$ They argued that the superior ORR/OER bifunctional activity of CoNi/NG is ascribed to the various active sites (i.e., $\mathrm{Co}-\mathrm{N}-\mathrm{C}, \mathrm{Ni}-\mathrm{N}-\mathrm{C}$ moieties, and $\mathrm{N}-\mathrm{C}$ sites) and its 2D graphene-like ultrathin structure as well as the synergistic effect of CoNi alloys. In addition, Mu's group demonstrated that the synergistic effect is substantial for electrocatalysis. ${ }^{43}$ The rational integration of the $\mathrm{N}$-doped carbon and $\mathrm{FeNi}_{3}$ intermetallic compound can effectively coordinate their advantages to enhance the catalysis performance. The cladding effect of N-doped carbon was reported to enhance the electrocatalytic performance of materials and effectively prevent the corrosion of catalysts in the catalytic process, exhibiting a good stability. ${ }^{43,44}$ For instance, Jiang's group developed a hybrid consisting of $\mathrm{Fe}_{3} \mathrm{C}$ and $\mathrm{Co}$ NPs encapsulated in $\mathrm{N}$-doped carbon with a nanoporous hierarchical structure via a template-removal method (denoted as $\mathrm{Fe}_{3} \mathrm{C}-\mathrm{Co} / \mathrm{NC}$ ) (Fig. 3b). ${ }^{45}$ Benefiting from the mesoporous carbon structure by combining $1 \mathrm{D}$ carbon nanotubes and $2 \mathrm{D}$ carbon nanosheets, and intimate integration between $\mathrm{Fe}_{3} \mathrm{C}$, Co, and $\mathrm{NC}$, the catalyst manifested a superior triple functional performance for the ORR, OER, and HER. The DFT results revealed that the $\pi$-bonds between $\mathrm{N}-\mathrm{C}$ and $\mathrm{C}-\mathrm{C}$ bonds were weakened with the help of $\mathrm{Fe}_{3} \mathrm{C}-\mathrm{Co}$, making the $\mathrm{OOH}^{*}$ and $\mathrm{H}^{*}$ bond to $\mathrm{C}-\mathrm{N}_{6}$ (the $\mathrm{C}$ atom adjacent to the pyridinic- $\mathrm{N}$ ) stronger, which will facilitate $\mathrm{OOH}^{*}$ formation and $\mathrm{H}^{+}$adsorption, i.e., more positive initial potentials for the ORR and HER in experiments. Furthermore, transition metal oxides/phosphides represent an appealing alternative owing to their wide availability, rich structural diversity, superior performance, and comparatively high corrosion resistance in a harsh environment. ${ }^{46}$ Based on theoretical calculations and experiments, Song's group reported hybrids of CoP and defective carbon as ORR and OER electrocatalysts, and revealed the interfacial charge polarization with the electrons gathering at the defective carbon surface and the holes gathering at the CoP surface due to the strong interfacial coupling. ${ }^{47}$ In addition, Masa et al. reported a carbon nanotubesupported tri-metallic ( $\mathrm{Mn}-\mathrm{Ni}-\mathrm{Fe}$ ) oxide as a bifunctional oxygen catalyst and revealed that the combination of the two components can influence the structural as well as catalytic properties, including the ORR/OER activity and ORR selectivity. ${ }^{48}$ Metal-nitrogen-carbon (M-N-C) catalysts have attracted much attention recently. The welldefined metal- $\mathrm{N}_{4}$ center in $\mathrm{M}-\mathrm{N}-\mathrm{C}$ catalysts is usually considered a possible active site for the ORR due to the appropriate adsorption energy of oxygen molecules. However, defects and metal- $\mathrm{N}_{4}$ sites are usually discussed separately, and the synergetic effect between them is hardly explored. Zhang's group synthesized atomic-scale $\mathrm{M}-\mathrm{N}-\mathrm{C}$ catalysts (denoted as FePc@N,P-DC) by directly coordinating the metal- $\mathrm{N}_{4}$ organic macrocyclic molecules $(\mathrm{FePc})$ with the $\mathrm{N}$-doped defective carbon nanosheets through a non-pyrolysis process (Fig. 3c). ${ }^{49}$ DFT calculations suggested that the covering FePc molecules decrease the overpotential of N,P-DC for the ORR. The enhanced ORR activities can be ascribed to the synergistic effect of highly conductive carbon nanosheets and the P-doped sites, leading to efficient electron transportation, optimizing the adsorption behaviors of the reaction zone, and tailoring the activity of the electrochemical catalysts. Liu's group reported a $\mathrm{Fe}_{x} \mathrm{~N}$ nanocrystal embedded $\mathrm{N}$-doped carbon material through post-treatment with $\mathrm{NH}_{3}$ and the $\mathrm{Fe}_{x} \mathrm{~N}$ nanocrystals can be modulated by altering the $\mathrm{NH}_{3}$ treatment time. ${ }^{50}$ The synergistic effects among the highly dispersed $\mathrm{Fe}_{x} \mathrm{~N}$ nanocrystals, well-doped nitrogen atoms, and hierarchical mesoporous structures boost their superior ORR catalytic activity and high methanol as well as carbon monoxide tolerance. Therefore, by taking full advantage of the synergistic effects of multiple active components in one integrated electrocatalyst, the electrocatalytic activity can be considerably enhanced due to the changes in the physicochemical properties and the electronic structure of materials. This finding opened a new avenue for designing a well-defined multifunctional electrocatalyst for renewable energy conversion and storage technologies.

\section{Structure-activity relationships}

DFT calculations can provide important insights and guidelines into understanding the structure-activity relationship and electrocatalytic mechanism, which are important for designing the material structure and constructing multiple active sites. Accurate catalyst structures are currently demonstrated by 
$\mathbf{a}$
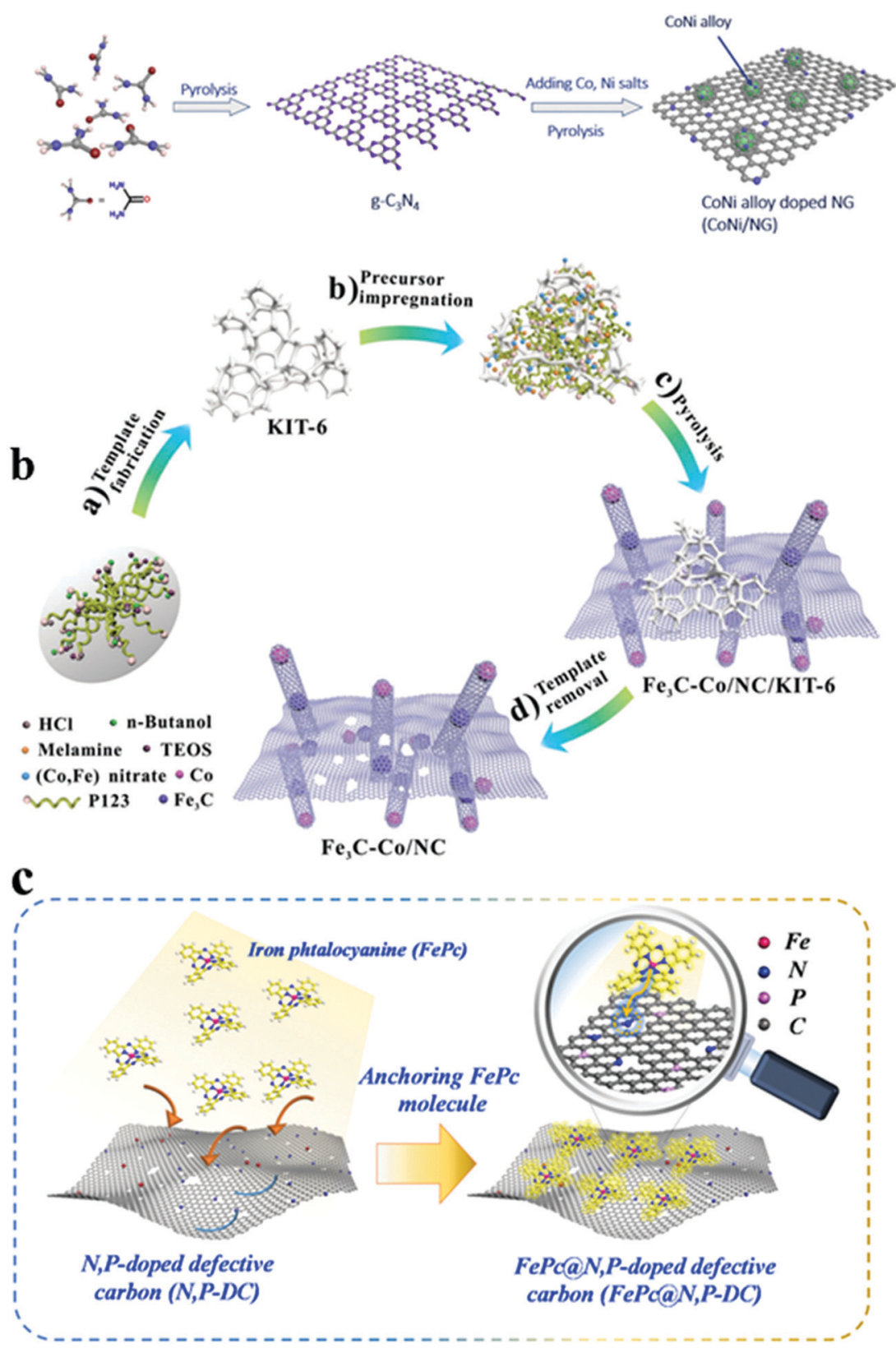

Fig. 3 Schematic illustration of the synthesis of carbon-coating metal-based NP hybrid materials. (a) CoNi/NG catalysts. Reproduced with permission from ref. 42. Copyright 2019, Elsevier. (b) Fe $3 \mathrm{C}-\mathrm{Co} / \mathrm{NC}$ catalysts. Reproduced with permission from ref. 45. Copyright 2019, Wiley-VCH. (c) FePc( N,P-DC catalysts. Reproduced with permission from ref. 49. Copyright 2020, Elsevier.

combining advanced in situ characterization and theoretical calculations. ${ }^{51-53}$ Thermodynamically, a better catalyst should be able to promote the ORR and OER with the overpotential being as low as possible. The free energy diagram and volcano plot are generally utilized to describe the ORR mechanism. Based on previous reports of Zhang's group, in terms of metalfree bifunctional catalysts, ORR and OER activity sites can be attributed to graphene edge effects and optimal edge N,P doping. ${ }^{24,25}$ In detail, the OER and ORR usually occur near the edge of the graphene but at different sites. Volcano plots (Fig. 4a and b) show that N,P-coupled doping can deliver the best OER performance $(0.39 \mathrm{~V})$, and the ORR overpotential could reach as low as $0.44 \mathrm{~V}$, which is superior to those of the benchmark Pt ( $\sim 0.45 \mathrm{~V}$ for ORR) and $\mathrm{RuO}_{2}(\sim 0.42 \mathrm{~V}$ for OER). The active site of the OER is located at the edge of the graphene, whereas that of ORR is the carbon that is situated near the $\mathrm{N}$-dopant in graphene edges (Fig. $4 \mathrm{c}-\mathrm{f}$ ). In addition, the DFT results manifested that the rate determining step (RDS) of the ORR is $\mathrm{OOH}^{*}$ formation, and the OER overpotential is reduced by the N,P-codoping (Fig. $4 \mathrm{~g}$ and $\mathrm{h}$ ). Despite numerous studies on the development of novel carbon-based catalysts, the ORR mechanism of the hybrid electrocatalyst with multiple active 
a

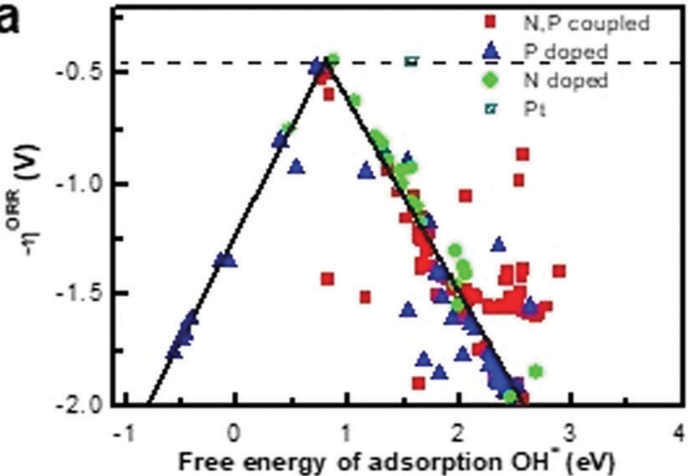

b
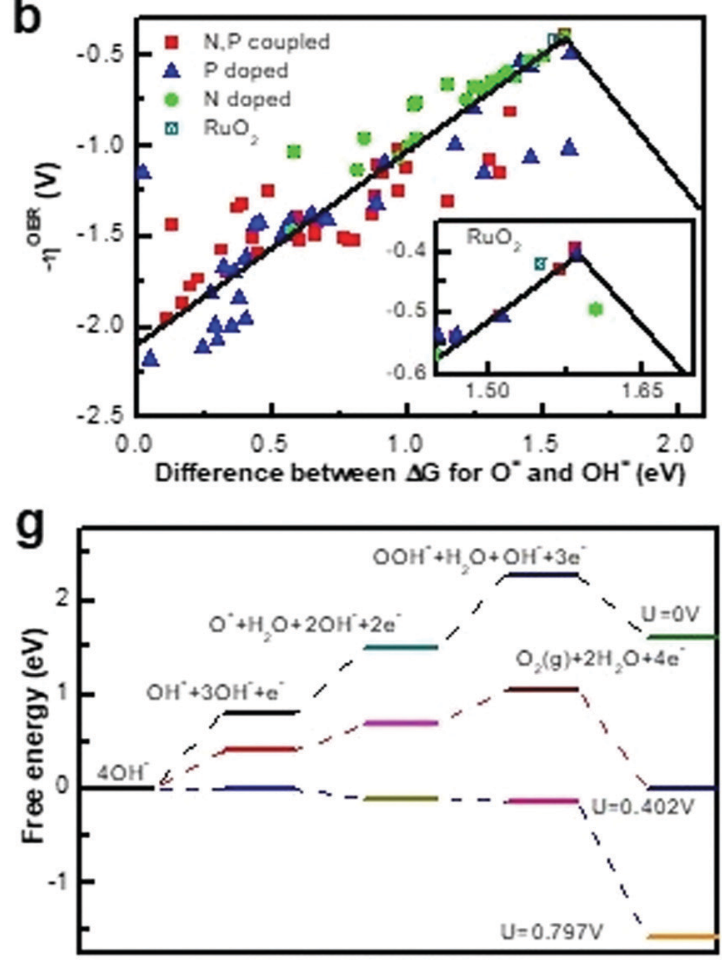

Reaction coordinate
C
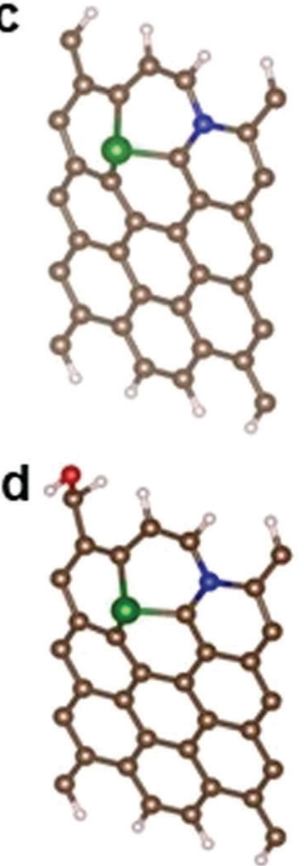

(1) $\mathrm{C}$ e

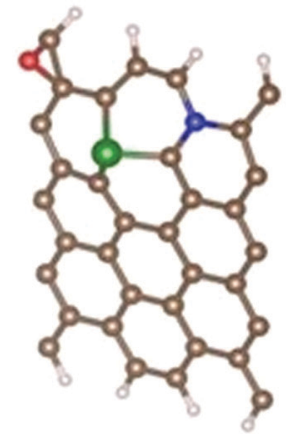

f

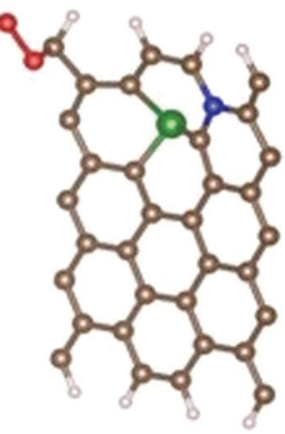

$\mathrm{H}$

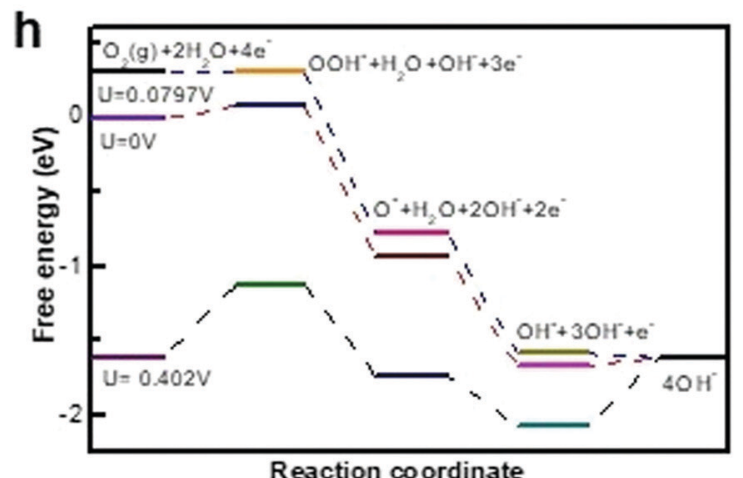

Reaction coordinate

Fig. 4 (a) Volcano plots of overpotential $\eta$ versus adsorption energy of $O^{*}$ of ORR. (b) Volcano plots of overpotential $\eta$ versus the difference between the adsorption energy of $\mathrm{O}^{*}$ and $\mathrm{OH}^{*}$ of OER. (c) Initial structure after the adsorption of hydroxyl $\mathrm{OH}^{*}$ (d) oxyl $\mathrm{O}^{*}$, and (e) peroxyl OOH*. (f) Intermediates on $\mathrm{N}$ and $\mathrm{P}$ coupled graphene. (g) Schematic energy profiles for the OER pathway and (h) ORR pathway. Reproduced with permission from ref. 24. Copyright 2015, Nature Publishing Group.

sites is not clear and consistent. Fu's group reported a Co nanoisland rooted on $\mathrm{Co}-\mathrm{N}-\mathrm{C}$ nanosheets supported by a carbon felt catalyst (denoted as $\mathrm{Co} / \mathrm{Co}-\mathrm{N}-\mathrm{C}$ ). ${ }^{54}$ DFT calculations were performed based on different active sites with the schematic models of $\mathrm{Co}(111)$, N-doped graphene, and $\mathrm{Co} / \mathrm{NC}$ (C site, N site, and Co site) to investigate the actual active sites. Fig. 5a shows that all adsorption energy values of $\mathrm{OH}^{*}, \mathrm{O}^{*}$, and $\mathrm{OOH}^{*}$ intermediates on the $\mathrm{C}$ sites of $\mathrm{Co} / \mathrm{NC}$ (blue) are much lower than those of the NC material, while the adsorption energy values of $\mathrm{OH}^{*}, \mathrm{O}^{*}$, and $\mathrm{OOH}^{*}$ intermediates on the Co site of $\mathrm{Co} / \mathrm{NC}$ are lower than those of pure Co. The $\mathrm{C}$ active sites in the $\mathrm{Co}-\mathrm{N}-\mathrm{C}$ structure play the key role in ORR activity, and the Co active sites play the key role in OER activity. In addition, the Co and $\mathrm{N}$ active sites in $\mathrm{Co} / \mathrm{NC}$ have the highest adsorption energy of the $\mathrm{OOH}^{*}$ intermediate, whereas the $\mathrm{C}$ active sites have the highest adsorption energy for the $\mathrm{O}^{*}$ intermediate, indicating that the RDS of the OER and ORR for the $\mathrm{Co}-\mathrm{N}-\mathrm{C}$ structure are the formation and decomposition of $\mathrm{OOH}^{*}$ and $\mathrm{O}^{*}$ intermediates. Furthermore, Peng's group synthesized a series of $\mathrm{Ni} / \mathrm{Co}$ ratio bimetallic coordination polymers using HITP (HITP $=2,3,6,7,10,11$-hexaiminotriphenylene) as the ligand to investigate the role of metal centers in modulating the ORR activity. ${ }^{55}$ Despite the decreased conductivity, $\mathrm{Co}_{3} \mathrm{HITP}_{2}$ exhibited excellent catalytic activities in the ORR and OER, which can be ascribed to the more active metal centers with unpaired $3 \mathrm{~d}$ electrons. Importantly, the ORR mechanism underwent a transition from the two-electron pathway on $\mathrm{Ni}_{3} \mathrm{HITP}_{2}$ to the four-electron pathway on $\mathrm{Co}_{3} \mathrm{HITP}_{2}$, which was successfully confirmed by DFT investigations on the intermediate binding energetics. Fig. 5b illustrates that all reaction intermediates 


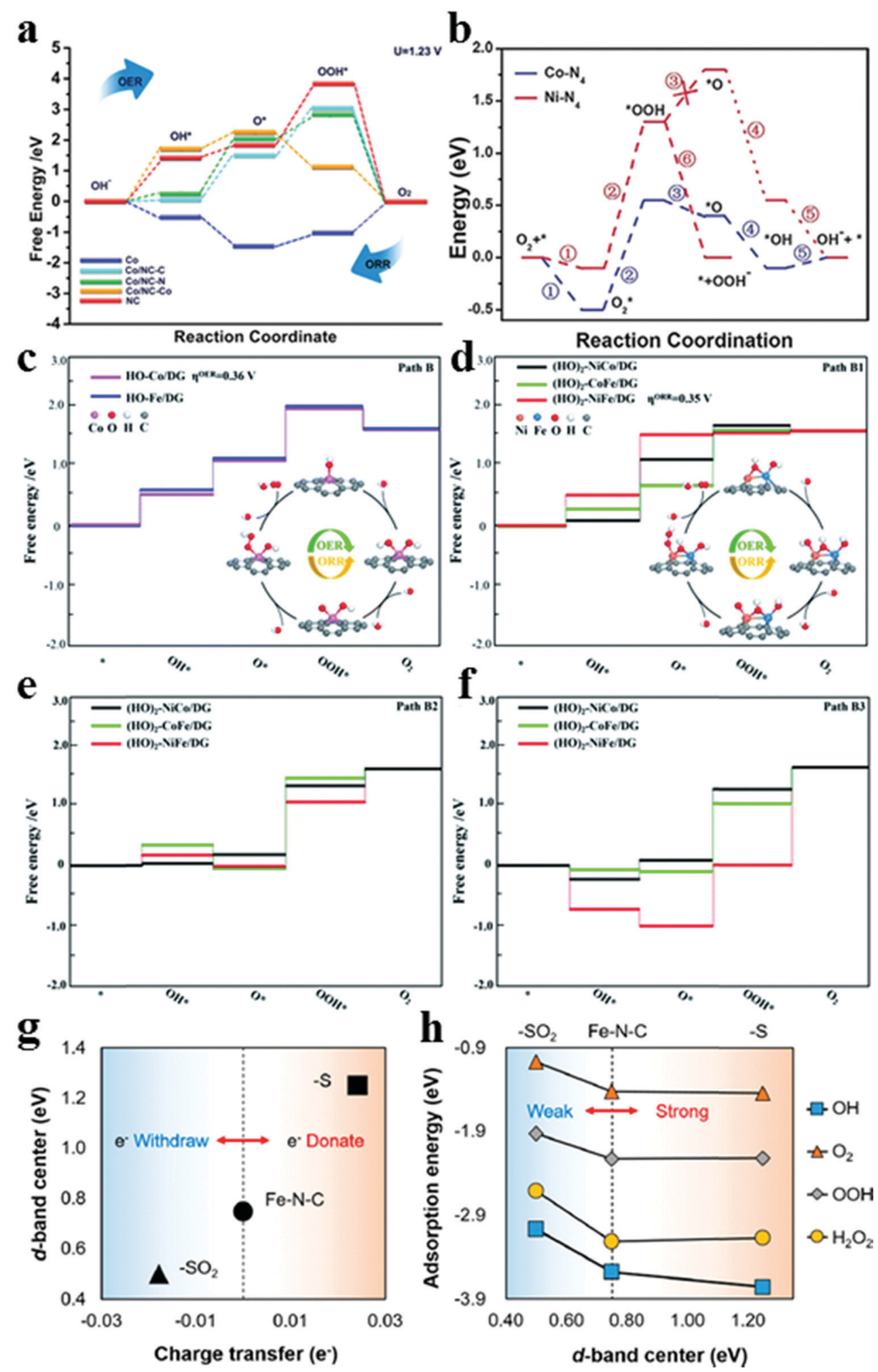

Fig. 5 (a) Calculated ORR/OER free energy diagram for $\mathrm{Co}, \mathrm{NC}$, and $\mathrm{Co} / \mathrm{NC}$ electrodes at various sites at the equilibrium potential (1.23 V) in an alkaline medium. Reproduced with permission from ref. 54. Copyright 2019, Wiley-VCH. (b) Free energy diagrams of intermediates along the reaction coordinates at a potential of $1.23 \mathrm{~V}$. Reproduced with permission from ref. 55. Copyright 2020, Wiley-VCH. Free energy diagrams of the OER and ORR with different $M$ centers. (c) $H O-M / D G$ (path B). (d) $(H O)_{2}-M^{1} M^{2} / D G$ (path B1). (e) $(H O)_{2}-M^{1} M^{2} / D G$ (path B2). (f) (HO) $2-M^{1} M^{2} / D G$ (path B3). Reproduced with permission from ref. 57. Copyright 2019, Royal Society of Chemistry. (g) Linear relationship between the amount of charge transfer from the functional groups and the $d$-band center. (h) Linear relationship between the adsorption energy of various intermediates and the $d$-band center. Reproduced with permission from ref. 58. Copyright 2019, American Chemical Society. 
$\left(\mathrm{O}_{2}{ }^{*}, \mathrm{OOH}^{*}, \mathrm{O}^{*}\right.$, and $\left.\mathrm{OH}^{*}\right)$ of the ORR exhibited a lower free energy on $\mathrm{Co}_{3} \mathrm{HITP}_{2}$ than on $\mathrm{Ni}_{3} \mathrm{HITP}_{2}$, suggesting a more strongly absorbed oxygen species with an improved thermodynamic stability. This finding could be due to the electron deficiency on the Co $3 \mathrm{~d}_{z^{2}}$ orbital of $\mathrm{Co}_{3} \mathrm{HITP}_{2}$, which can enhance the metal center activity. The authors also presented that the unpaired electron on Co $3 \mathrm{~d}_{z^{2}}$ within a metastable quadrilateral coordination can boost the activity of the metal center, the binding of oxygen species, and ultimately lead to enhanced ORR activities. Thermodynamic analysis based on theoretical limiting potentials is fundamental in rationalizing the activity trends for metals and guiding the design as well as optimizing various catalysts. However, the rates of the reaction and electrochemical barriers play a key role in the ORR current, and the effect of kinetics on the ORR should be considered. Zhao's group reported that a prototype study of (Co and/or Fe)$\mathrm{N}_{x}(x=1-6)$ embedded configurations (FeCoN ${ }_{x}$-gra) was conducted within DFT via spin-polarized calculations. ${ }^{56}$ The results indicated that an itinerant ferromagnetic half metal can be considered as a promising alternative for the ORR and OER. The ferromagnetically coupled spin-polarized electrons in a half metal could attract oxygen molecules, and thus stimulate the ORR, while the formation of the $\mathrm{O}-\mathrm{O}$ bond in the OER requires spin conservation. Typically, the metallic spin of a half metal could moderate the binding strength of the main reaction intermediates. Furthermore, Pei's group utilized hydroxyl group modified single- and bi-metal atoms supported on defective graphene (DG) to study the electrocatalytic ORR and OER under alkaline conditions. ${ }^{57}$ The DFT calculation results indicated that the hydroxyl-group-modified single- and bi-metal atomic catalysts (M/DG, $\mathrm{M}=\mathrm{Ni}$, Fe and Co; $\mathrm{MM} / \mathrm{DG}$, where $\mathrm{M}$ is $\mathrm{Ni}$, Co, and Fe; $\mathrm{M}^{1} \mathrm{M}^{2} / \mathrm{DG}$, where $\mathrm{M}^{1}$ and $\mathrm{M}^{2}$ are Ni, Co, and $\mathrm{Fe}$, respectively) could promote bifunctional oxygen activities compared with the bare atomic metal catalyst, which can be attributed to the high valence of the transition metal atoms. Fig. 5c shows that the transformation from $\mathrm{O}^{*}$ to $\mathrm{OOH}^{*}$ is the potential-determining step on the $\mathrm{HO}-\mathrm{Co} / \mathrm{DG}$ and $\mathrm{HO}-\mathrm{Fe} / \mathrm{DG}$ for the OER, and overpotentials are measured to be 0.36 and $0.39 \mathrm{~V}$, respectively. In reverse ORR, the $\mathrm{OOH}^{*}$ formation step is the potential-determining step. Two kinds of reaction centers are on the hydroxylated bi-atomic catalysts, namely, singletransition-metal atom center $\left(\mathbf{M}, \mathbf{M}^{1}\right.$, and $\left.\mathbf{M}^{2}\right)$ and bi-metal atom center $\left(\mathbf{M}-\mathbf{M}\right.$ and $\left.\mathbf{M}^{1}-\mathbf{M}^{2}\right)$. Fig. $5 \mathrm{~d}-\mathrm{f}$ show that the Co center of CoFe/DG exhibited the highest OER activity, and the $\mathrm{Ni}$ center of NiFe/DG exhibited the highest ORR activity. The Fe atom can effectively regulate the electronic structure of $\mathrm{Ni}$ and Co atoms on hydroxylated CoFe/DG and NiFe/DG, leading to the enhanced ORR and OER activity of $\mathrm{Ni}$ and Co atoms. In addition, Lee's group presented a versatile strategy for tuning the ORR activity of a single $\mathrm{Fe}-\mathrm{N}_{4}$ site by controlling the electronwithdrawing/donating properties of the carbon matrix. ${ }^{58}$ They found that the introduction of oxidized sulfur (S) could decrease the d-band center of Fe by withdrawing electrons, thus facilitating $\mathrm{ORR}$ at the $\mathrm{Fe}-\mathrm{N}_{4}$ site by lowering the intermediate adsorption energy. Fig. $5 \mathrm{~g}$ shows that the half-wave potentials of FeNC-SMSUFC catalysts showed a tendency similar to that of the ratio of oxidized S and thiophene-like $\mathrm{S}$. This finding suggested that the change in the ORR activity of the $\mathrm{Fe}-\mathrm{N}_{4}$ sites was due to the electronic effect of the $\mathrm{S}$ functionalities incorporated in the carbon plane. The DFT calculated d-band center was up-shifted with the increasing amount of charge transfer from the functional group to the $\mathrm{Fe}-\mathrm{N}_{4}$ site (Fig. 5h), which can induce a strong interaction between the $\mathrm{Fe}-\mathrm{N}_{4}$ site and the adsorbate. Although nitrogendoped carbon-based materials have been demonstrated as efficient ORR/OER catalysts, which type of $\mathrm{N}$ dopant being conducive to the enhanced catalytic performance still requires systematic exploration. To this end, Zhao's group reported X-doped ( $\mathrm{X}=\mathrm{B}, \mathrm{N}, \mathrm{P}$, and $\mathrm{S}$ ) graphdiynes via DFT computations. ${ }^{59}$ The result revealed that $\mathrm{sp}-\mathrm{N}$ doped graphdiynes, graphitic S-doped, and graphitic P-doped graphdiynes exhibit comparable or even better ORR/OER catalytic performance than $\mathrm{Pt} / \mathrm{C}$ or $\mathrm{RuO}_{2}$. The sp-N dopant is the most crucial dopant for $\mathrm{N}$-doped graphdiynes. It is illustrated that the $\mathrm{C}$ atoms adjacent to $\mathrm{N}$ - and P-dopants and possessing high spin are the OER active sites, whereas those adjacent to $\mathrm{N}$ - and S-dopants and featuring large positive charges are the ORR active sites. These results can provide a specific link between the electron structure and electrocatalytic performance which provides valuable information for catalyst design.

\section{Bifunctional carbon-based catalysts}

The synthesis of carbon-based catalysts mainly depends on high-temperature pyrolysis, and their intrinsic activity is attributed to the type of active site. ${ }^{60}$ In addition, different precursors can be divided into traditional carbon materials and metal-organic framework (MOF)-derived carbon-based materials. For bifunctional carbon-based electrocatalysts, the recently reported active materials are heteroatom doped carbon, carbon-supported non-noble metal composites, metal-nitrogen-carbon (M-N-C), etc. In the following sections, the discussion of the design of carbon-based active materials will focus on these three aspects.

\subsection{Heteroatom-doped carbon active sites}

Due to the different electronegativities between the heteroatoms, such as N, F, S, O, P, and C atom, the doping-induced charge or spin redistribution can regulate properly the adsorption energy for reactant/reaction intermediates. ${ }^{61-63}$ DFT calculations indicate that heteroatom-doping is a key origin of the activity for heteroatom-doped carbon electrocatalysts. ${ }^{24,25}$ Indeed, a nitrogen and phosphorous co-doped carbon sphere (NPCS) catalyst was prepared by using manganese dioxide spheres as both the redox initiator and the self-sacrificing template for the in situ interfacial polymerization of aniline. After pyrolysis, the obtained highly porous doped electrocatalysts manifested the $\mathrm{Pt} / \mathrm{C}$-comparable ORR performance with a half-wave potential (0.83 V vs. RHE) and long-term charging-discharging stability (337 cycles) in a Zn-air battery (Fig. 6a). It was firstly revealed that the edge effect to expose more active sites around the edges is crucial to achieve the high performance for rechargeable batteries on the basis of the experimental results and theoretical calculations. ${ }^{25}$ Recently, Zhou et al. proposed a three-dimensional 


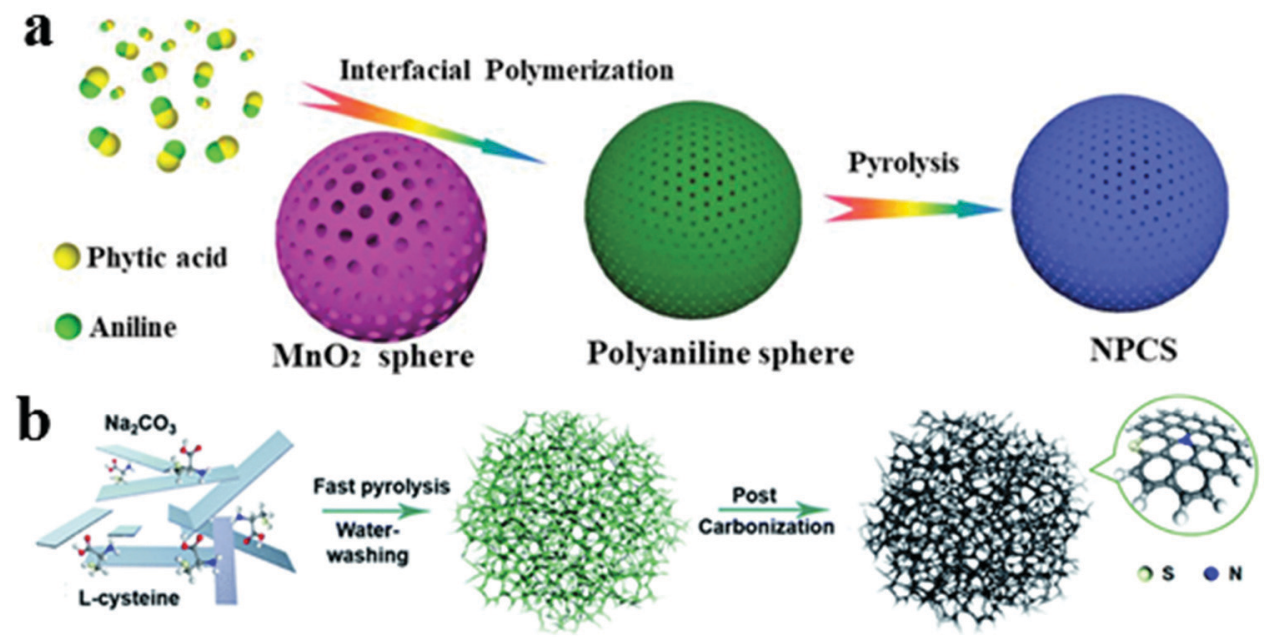

Fig. 6 (a) Schematic illustration of the preparation of NPCS. Reproduced with permission from ref. 25. Copyright 2019, Elsevier. (b) Schematic illustration of the preparation of SNCs. Reproduced with permission from ref. 64. Copyright 2020, Royal Society of Chemistry.

(3D) porous $\mathrm{S}$ and $\mathrm{N}$ co-doped carbon nanosheets (SNCs) by a simple two-step heat treatment with L-cysteine as the $\mathrm{C}, \mathrm{N}$ and $\mathrm{S}$ source, respectively, as illustrated in Fig. $6 \mathrm{~b} .{ }^{64}$ With high nitrogen and sulfur atomic content of 4.92 and $1.54 \%$, DFT calculations revealed that the introduction of $\mathrm{S}$ into graphitic $\mathrm{N}$ doped graphene can decrease the OER overpotential obviously. The improvement of catalytic performance is attributed to the synergistic effect of $\mathrm{N}$ and $\mathrm{S}$.

\subsection{Carbon-supported non-noble-metal-based active sites}

Zero-dimensional carbon quantum dots, 1D carbon nanotubes, 2D graphene, and their derivatives featuring excellent electrical conductivity as well as abundant edges active sites have been widely investigated. ${ }^{13,65}$ In addition to direct utilization as electrocatalysts, carbon nanomaterials can widely serve as the catalyst support. ${ }^{66}$ Numerous experimental studies have proven that defective carbon and/or heteroatom-doped carbon are necessary for metal loading. ${ }^{67}$ To date, the enhancement of bifunctional carbon-based catalyst activity has two directions: (1) improving the intrinsic catalytic activity of each active site by modulating the composition of catalysts, such as combining various transition metals/alloys, metal oxides, nitrides, carbides, phosphides, and chalcogenides and (2) improving the number of active sites by tuning the geometric structure and size to expose more active sites and facilitate mass transfer. In general, these two strategies are often applied simultaneously. Increasing mass loading is one available method to improve the activity, but the intrinsic activity is dominant. In this regard, integrating highly reactive transition metals with highly conductive carbon materials to achieve maximum activity and costs effectiveness is acceptable. For example, Xing's group synthesized catalysts featured with thin graphene nanosheet coupling with full encapsulated ultrafine and highly loaded $(\sim 25 \mathrm{wt} \%)$ transition metal NPs (denoted as TMs@NCX) by optimizing the electronic modulation effect from suitable metal cores for the ORR and OER (Fig. 7a). ${ }^{68}$ High-resolution TEM images revealed that TMs were well-encapsulated by 3-5 graphitic layers, the NiFe core were $\mathrm{Ni}$ and Fe mixed at the atomic level, and the lattice fringe of $2.06 \AA$ corresponded to the (111) plane in the cubic $\mathrm{NiFe}_{2}$ phase (Fig. $7 \mathrm{~b}$ and c). Surface graphitic layers can be adjusted by an electronic modulation effect from suitable metal cores and have a positive effect on intrinsic activity. This work confirmed that the synergistic effect of advantageous metal-substrate interaction can generate efficient active sites and considerably improve the activity and stability of the metal supported on carbon materials. Recent studies suggested that amorphous metal oxides show excellent performance owing to the increased number of active sites from lattice defects and/or improved ionic conductivity due to their abundant oxygen vacancies. ${ }^{69,70}$ Graphene with large specific surface areas as conductive substrates is beneficial for increasing the electrical conductivity and accommodating more active bimetallic oxide catalysts in the preparation of highly efficient heterogeneous electrocatalysts. Chen's group reported hybrid materials comprising amorphous bimetallic oxide NPs anchored on N-doped reduced graphene oxide (denoted as $\mathrm{Fe}_{a} \mathrm{Co}_{1-a} \mathrm{O}_{x} / \mathrm{NrGO}$ ) (Fig. 7d). ${ }^{71}$ The amorphous characteristics of the $\mathrm{Fe}_{0.5} \mathrm{Co}_{0.5} \mathrm{O}_{x}$ can be proved by X-ray diffraction patterns. The potential difference of the ORR and OER in $0.1 \mathrm{M}$ $\mathrm{KOH}$ electrolyte is typically $0.78 \mathrm{~V}$, demonstrating an excellent bifunctional catalytic activity. In a more recent research, the transition metal-containing $\mathrm{N}$-doped carbon materials could greatly improve the graphitization and electrical conductivity of the carbon matrix, preventing corrosion and aggregation during electrochemical reactions. Furthermore, interactions and synergistic effects between metal species and N-doped carbon species enhance the intrinsic electrochemical activity of the ORR and OER by electronically modulating the charge distribution on active sites and the adsorption/desorption of $\mathrm{O}_{2}$ as well as intermediates. ${ }^{72}$ $\mathrm{Hu}$ 's group reported a 3D hierarchical $\mathrm{Co}-\mathrm{N}-\mathrm{C}$ brush-like nanostructure by N-doped carbon encased Co NPs by selfcatalyzed growth (denoted as CoNC-NB) (Fig. 7e). ${ }^{72}$ The obtained catalyst presented a higher surface area, more accessible active sites, and enhanced diffusion kinetics. In this case, the rechargeable $\mathrm{Zn}$-air battery exhibited a high peak power density of $246 \mathrm{~mW} \mathrm{~cm}^{-2}$ 
$\mathbf{a}$

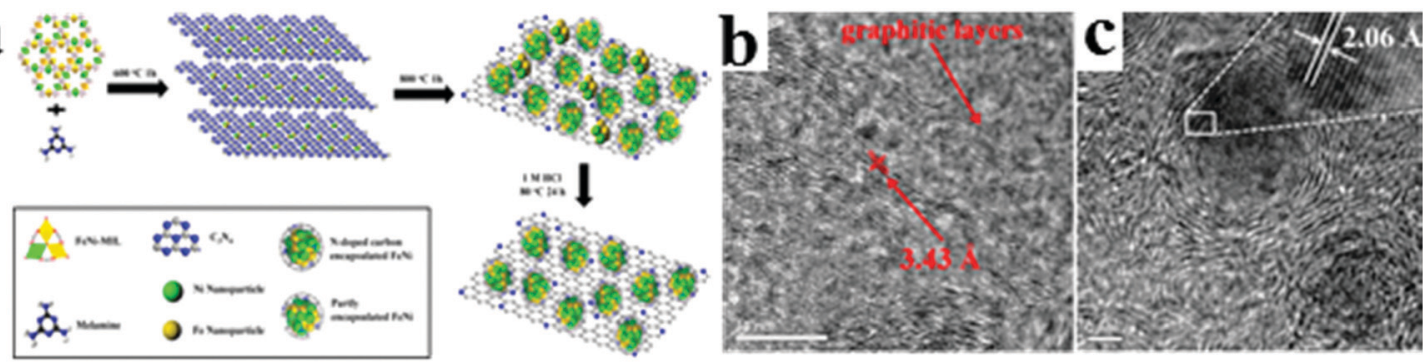

d
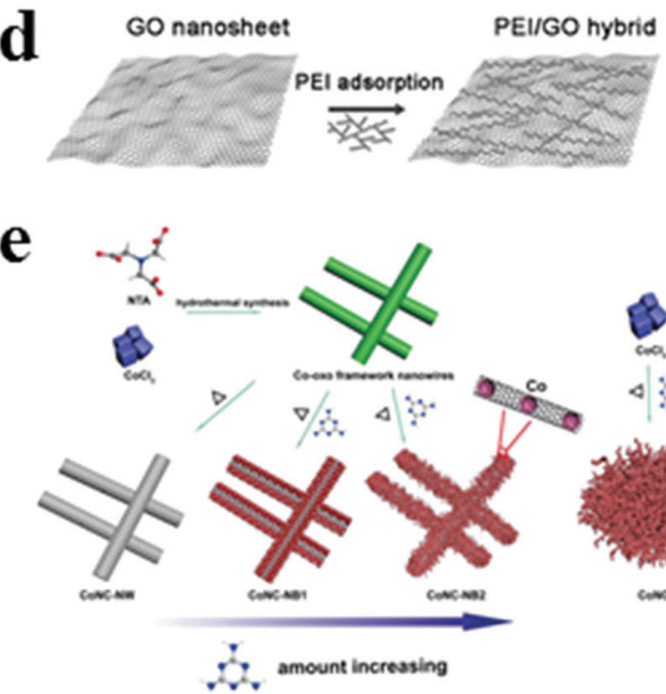
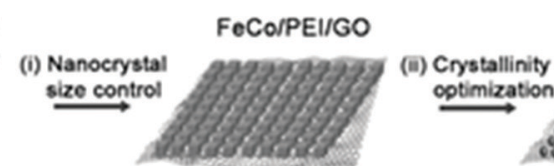

$\mathrm{Fe} . \mathrm{C} 01, \mathrm{O} / \mathrm{NrGO}$ (iii) Composition optimization

\section{f}

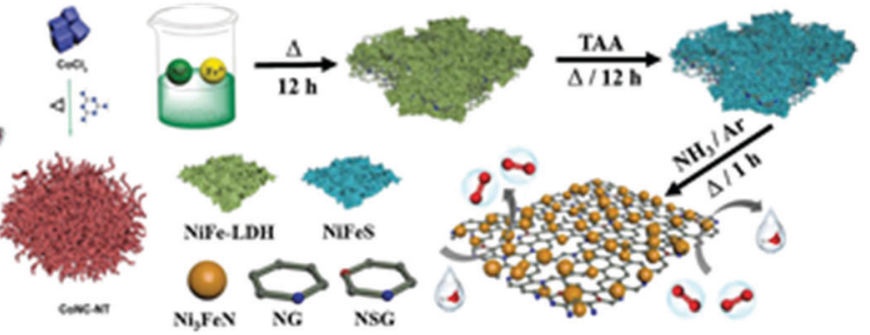

Fig. 7 (a) Schematic illustration of the synthetic strategy of the TMs(aNCX composite. (b) HR-TEM images of NiFeaNCX. (c) Crystal (111) plane of the NiFe alloy. Reproduced with permission from ref. 68. Copyright 2016, American Chemical Society. (d) Schematic illustration of the synthesis of $\mathrm{Fe}_{a} \mathrm{CO}_{1-a} \mathrm{O}_{x} / \mathrm{NrGO}$ hybrid catalysts. Reproduced with permission from ref. 71. Copyright 2017, Wiley-VCH. (e) Schematic illustration of the preparation of various $\mathrm{Co}-\mathrm{N}-\mathrm{C}$ nanostructures. Reproduced with permission from ref. 72. Copyright 2020, Wiley-VCH. (f) Schematic illustration for the synthesis of $\mathrm{S}-\mathrm{Ni}_{3} \mathrm{FeN} / \mathrm{NSG}-700$. Reproduced with permission from ref. 74. Copyright 2020, Elsevier.

and a superior long cycling stability. In addition, experimental evidence indicated that the dual heteroatom decorative NiFebased carbon materials, including NiFe oxides/nitrides, can substantially regulate the electronic structure and induce proper distorted structures of transition metals to optimize the intermediate adsorption and improve the ORR activity compared with NiFe oxide/hydroxides when applied for $\mathrm{Zn}$-air batteries. ${ }^{73}$ For instance, Wang's group recently reported $\mathrm{S}$ modulated $\mathrm{Ni}_{3} \mathrm{FeN}$ NPs supported on N/S co-doped graphene with a porous structure as Zn-air battery and water splitting device catalysts (denoted as $\mathrm{S}-\mathrm{Ni}_{3} \mathrm{FeN} / \mathrm{NSG}$ ) (Fig. 7f). ${ }^{74}$ The DFT calculations revealed that the $\mathrm{S}$ decoration can effectively promote the formation of $\mathrm{OOH}^{*}$ intermediates and decrease the Gibbs free energies for the OER. Benefiting from the increase of accessible highly active sites and enhanced mass transfer and electron transportation, the optimal catalyst exhibits superior electrocatalytic durability. The number of active sites is strongly dependent on a crucial factor, namely, dispersity. ${ }^{75}$ An ideal catalyst has effective active sites, enhanced effective surface area, and mass transfer rate. Nanoscale size catalysts usually suffer from uncontrollable aggregation in the high-temperature pyrolysis and the peeling phenomenon in the catalytic process, which leads to a tremendous decrease in electrocatalytic activity. Zhang's group took full advantage of the natural organic molecules that featured abundant functional groups and stable macrocyclic structure, and made them anchoring agents and dispersants for metal ions to obtain a series of bifunctional catalysts with a high dispersibility and an excellent activity. ${ }^{39,40,76}$ For example, we synthesized N, S co-doped porous carbon with interspersed hollow cobalt oxide NPs. ${ }^{40}$ Cobalt ions were efficiently and uniformly bound on the polymer chain owing to the polyethyleneimine molecule with multiple amine groups and good coordination ability. Coincidentally, to optimize the geometry structure further and obtain a superior zinc-air battery cathode catalyst, we utilized tannic acid with abundant phenolic hydroxyl and strong chelating ability to coordinate metal ions, constructing a multifunctional catalyst of $\mathrm{N}$-doped graphene embedded with $\mathrm{Co}_{5.47} \mathrm{~N}$ NPs $\left(\mathrm{CO}_{5.47} \mathrm{~N} @ \mathrm{~N}-\mathrm{rGO}\right) .{ }^{39}$ Moreover, this crystal structure of the $\mathrm{Co}_{5.47} \mathrm{~N}$ phase possesses several nitrogen vacancies, which is beneficial for ensuring the metallic properties of $\mathrm{Co}_{5.47} \mathrm{~N}$ and enhances the electrical conductivity. Tannic acid plays an essential role in regulating the microscopic morphology and phase composition. The 2D layer structure of graphene was well maintained after the addition of cobalt ions with the assistance of tannic acid, allowing ammonia gas to diffuse into the inner space easily and combine with cobalt species to form cobalt nitride rather than metallic cobalt. As expected, the $\mathrm{Co}_{5.47} \mathrm{~N} @ \mathrm{~N}-\mathrm{rGO}-750$ exhibited excellent electrocatalytic activities toward the ORR, HER, and OER, 
and the water splitting driving by $\mathrm{Zn}$-air batteries exhibited good gas generation rates. As per previous reports, polypyrrole (PPy) nanotubes can be a desirable choice to hold the post of a template and nitrogen source owing to their special hollow structure and high nitrogen content. Recently, Chen's group developed Co NPs embedded in hollow N-doped carbon tubes (Co@hNCTs) by using PPy as the template. ${ }^{77}$ Benefiting from the interconnected porous conductive framework and uniformly dispersed Co NPs embedded within the PPy skeleton, the synthesized catalysts manifested a better catalytic selectivity and stability toward the ORR with a halfwave potential of $0.87 \mathrm{~V}$.

\section{$5.3 \mathrm{M}-\mathrm{N}-\mathrm{C}(\mathrm{M}=\mathrm{Fe}, \mathrm{Co}, \mathrm{Ni}, \mathrm{Cu})$ active sites}

Metal-organic frameworks are a class of materials constructed by connecting metal ions or clusters with organic linkers, exhibiting open crystalline structures, exceptional porosity, structural flexibility, and tunable functionality. Porosity plays an important role in ion diffusion and mass transfer. An excellent catalyst requires not only effective active sites but also a porous structure to facilitate reactant migration. In general, MOF-derived carbon materials also depend on the thermal pyrolysis of MOF precursors. ${ }^{60}$ For instance, Guan et al. reported a Co single atom electrocatalyst assembled in $\mathrm{N}$-doped porous carbon nanoflake arrays (denoted as NC-Co SA), which was fabricated by a facile carbonization-acidification using metal MOFs as the precursor material. ${ }^{78}$ Compared with the Co NP counterpart (denoted as NC-Co), the NC-Co SA showed a considerably enhanced ORR and OER performance. Hard and soft templates are commonly used to obtain carbon materials with a controlled porosity. Changing the MOF synthesis conditions, introducing templates in MOF synthesis, MOFs with various morphologies, including 0D polyhedral and nanospheres; 1D nanorods, nanowires, and nanotubes; 2D nanosheets; 3D arrays; and other hierarchical structures, have been reported in the synthesis of carbon-based materials with morphologypreserved transformations by selecting different types of MOFs. ${ }^{52,79,80}$ In addition, MOF-derived carbon materials consisting of metal-based NPs, ${ }^{9,81}$ and atomically dispersed non-precious metal nitrogen-carbon ( $\mathrm{M}-\mathrm{N}-\mathrm{C}, \mathrm{M}=\mathrm{Fe}, \mathrm{Co}, \mathrm{Ni}$, and $\mathrm{Cu}$ ) active sites were prepared. ${ }^{52,53,80,82} \mathrm{M}-\mathrm{N}-\mathrm{C}$ catalysts have emerged as promising electrocatalysts for electrocatalysis and energy storage but also face challenges, including (1) poor utilization of active species and (2) unclear catalytic active sites. ${ }^{79,83} \mathrm{M}-\mathrm{N}-\mathrm{C}$ catalysts with atomic dispersion especially derived from the MOFs have also attracted great attention owing to their highly dispersed active species and satisfactory electrochemical performance. ${ }^{84}$ For example, Jiang's group recently reported well-aligned single cobalt atom catalysts derived from urchin-like nanotube structures ZIF-67 (denoted as UNT SAs/N-C), as is displayed in Fig. $8 \mathrm{a}^{51} \mathrm{X}$-Ray absorption near-edge spectroscopy (XANES) and extended X-ray absorption fine structure results revealed that the single Co atoms have a $\mathrm{CoN}_{4}$ coordination structure in UNT SAs/N-C. Fig. 8b shows that the maximum value of wavelet transform intensity is near $3.6 \AA^{-1}$, indicating the formation of $\mathrm{CoN}_{4}$ coordination pattern in UNT Co SAs/N-C. Additionally, the DFT calculation results showed that the electron transfer in $\mathrm{CoN}_{4}$ is much easier to implement within the applied potential, thus leading to a better catalytic performance toward the ORR/OER. The polyhedron is one of the most common morphologies of MOF particles, consisting of zeolite imidazolate frameworks (ZIFs) and UiO, MIL series. Although they have been used as precursors to synthesize carbon polyhedra through thermal conversion, monometallic atomically dispersed $\mathrm{M}-\mathrm{N}-\mathrm{C}$ capabilities toward the OER are unsatisfactory, bringing a great challenge for the realization of an efficient ORR/OER bifunctional catalysis. The chemical interaction and synergistic effect of heterogeneous bimetallic species for boosting the reversible ORR/OER remain unclear. All these factors are the foundations of rationally designed SACs with optimized composition and structure as highly efficient, durable functional catalysts for chemical and electrochemical reactions. Thus, Hu's group reported novel atomically dispersed binary Co-Ni sites embedded in $\mathrm{N}$-doped hollow carbon nanocubes (denoted as CoNi-SAs/NC) by pyrolyzing the dopamine (DPA)-coated MOFs (Fig. 8c) ${ }^{85}$ Fig. 8d shows the isolated single-metal atoms in HAADF-STEM. Synchrotron X-ray absorption spectroscopy measurements were performed to identify the atomic and coordination configurations in synthesized samples further. Fig. 8e manifests that the near-edge absorption energy of the CoNiSAs/NC is higher than that of CoNi-NPs/NC, implying that the Co single atoms are more positively charged in CoNiSAs/ NC. As expected, the first major peak for CoNi-SAs/NC at $\approx 1.49 \AA$ corresponds to the dominant $\mathrm{Co}-\mathrm{N}$ coordination that has been widely recognized in SACs (Fig. 8f). Furthermore, a second peak at $\approx 2.22 \AA$ could be assigned to the metal-metal coordination, which cannot be found in pure $\mathrm{Co}-\mathrm{N}-\mathrm{C}$ and $\mathrm{Ni}-\mathrm{N}-\mathrm{C}$, proving the formation of a diatomic Co-Ni coordination. The enhanced ORR and OER electrocatalytic activity could be attributed to the abundant single-atom active sites as well as the synergistic effect from the diatomic structure in porous conductive carbon frameworks, thus reducing the overpotential and facilitating electron transfer. Furthermore, the synergistic interaction of a dual metal-N structure (Co-Ni-N) can be demonstrated by DFT calculations. The synergistic interaction of dual metal-N structures $(\mathrm{Co}-\mathrm{Ni}-\mathrm{N})$ can decrease the energetic barrier and accelerate the reaction kinetics compared with monometallic $\mathrm{Ni}-\mathrm{N}$ and CoNi NP counterparts. When the CoNi-SAs/NC was applied as the cathode catalyst, the solid flexible rechargeable Zn-air battery exhibited a stable discharge and charge performance (Fig. 8g), demonstrating an excellent flexibility. Wu's group recently demonstrated a surfactant-assisted MOF approach to prepare a series of core-shell structure atomically dispersed Co-doped carbon catalysts. ${ }^{86}$ The size and morphology of the catalysts mainly rely on the surfactant species (Fig. 8h). The confinement effect from the cohesive interactions between the surfactant and the ZIF-8 nanocrystals plays an essential role of suppressing the agglomeration of Co atomic sites and mitigating the collapse of internal microporous structures of ZIF-8. Among the studied surfactants, the pluronic F127 block copolymer exhibits the greatest performance due to having double the active site density compared to that of the surfactant free catalyst. DFT calculations show that unlike other Co catalysts, this new atomically dispersed Co-N-C@F127 catalyst is believed 
$\mathbf{a}$

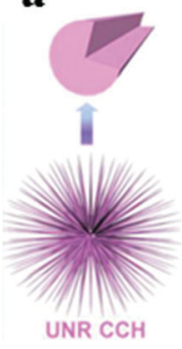

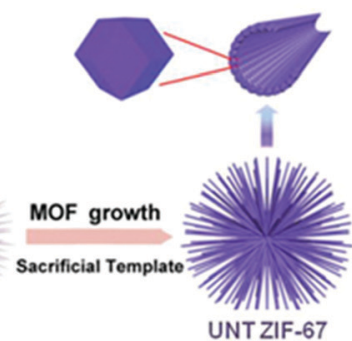
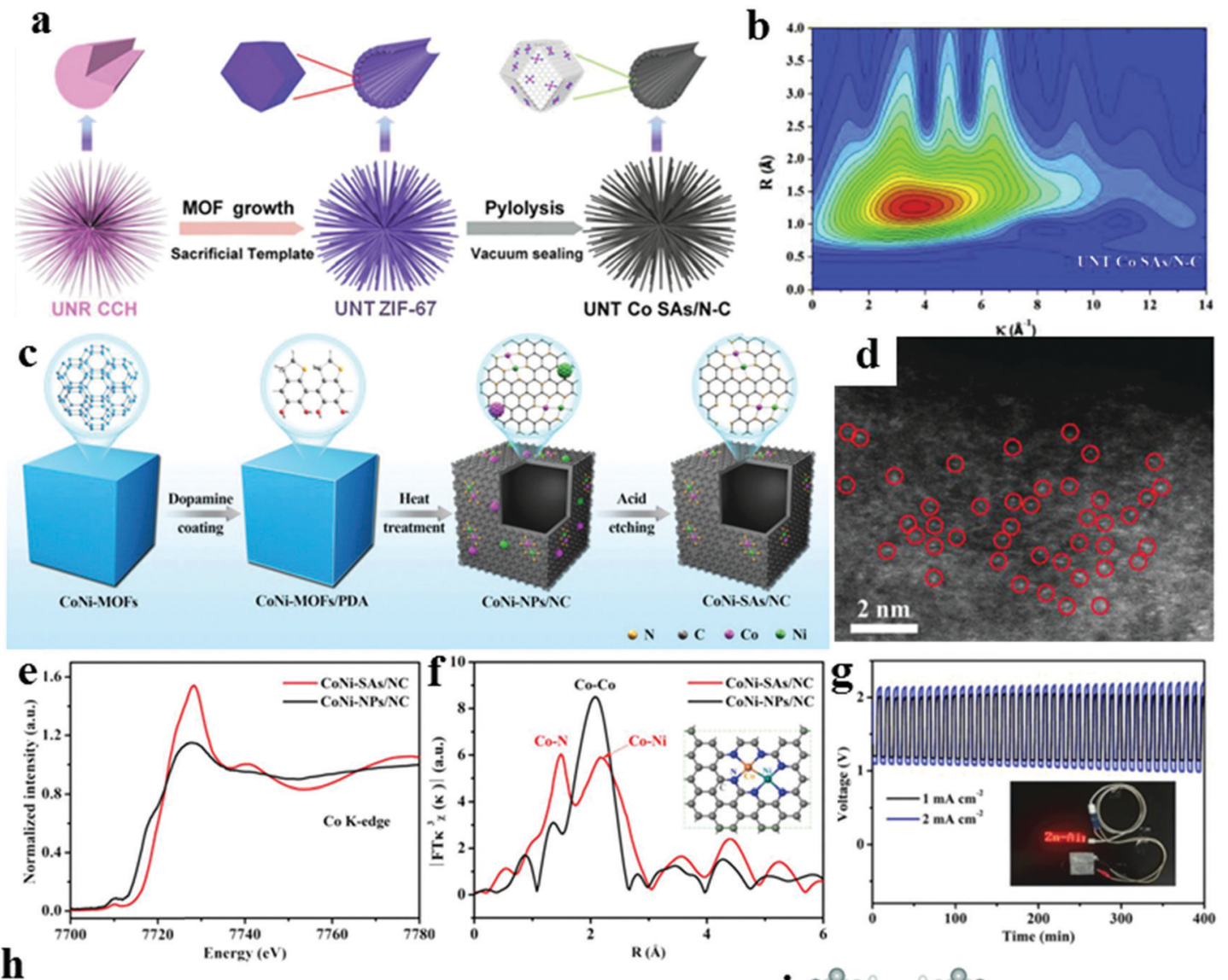

h
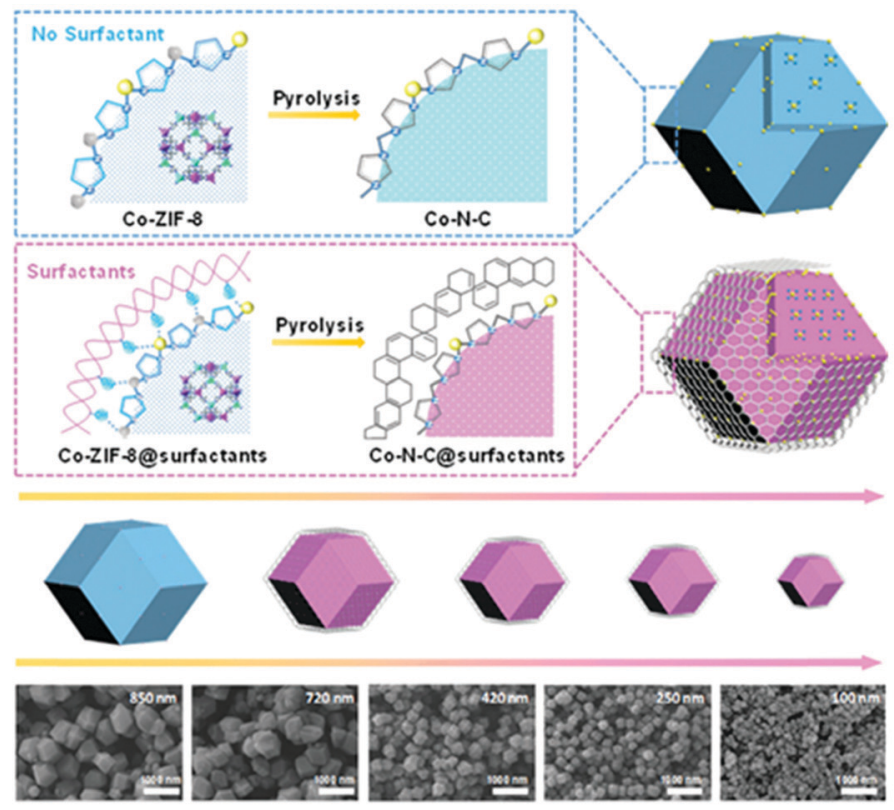

Non-s urfactant

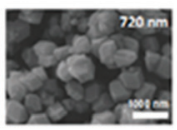

SOS
$(M w=288 \mathrm{~g} / \mathrm{m}$

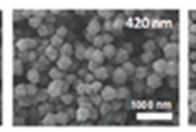

CTAB

CTAB
(Mw $=364 \mathrm{~g} / \mathrm{mol})$

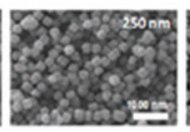

F127
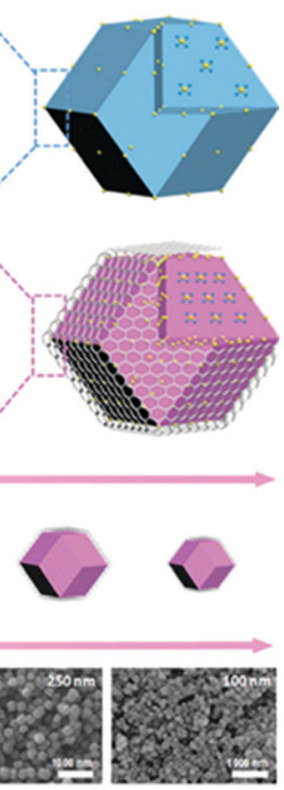

PVP

PVP
$40,000 \mathrm{~g} / \mathrm{mol}$ i $0^{\circ}$

o. 00.000 0.0000 $0.0 \%$ $\begin{array}{cc}0.0 & 0 \\ 0.0 & 0.0 \\ 0.0 & 0.0\end{array}$

$\mathrm{CoN}_{2+2}$

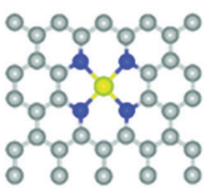

\section{j}

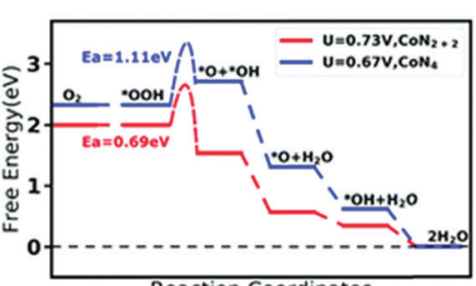

$\mathbf{k}$

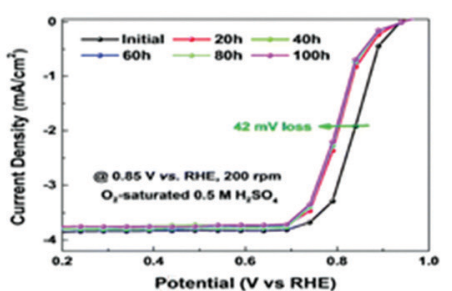

Fig. 8 Schematic illustration of the synthesis of $M-N-C$ sites in MOF-derived carbon-based materials. (a) Hierarchical UNT Co SAs/N-C catalysts. (b) Wavelet transform of UNT Co SAs/N-C. Reproduced with permission from ref. 51. Copyright 2019, Elsevier. (c) CoNi-SAs/NC. (d) HAADF-STEM of CoNi-SAs/NC. (e) Co K-edge XANES spectra. (f) Fourier transforms of Co K-edge spectra of CoNi-SAs/NC and CoNi-NPs/NC. (g) Charge and discharge cycling stability. Reproduced with permission from ref. 85. Copyright 2019, Wiley-VCH. (h) Core-shell-structured Co-N-C@surfactant catalysts and SEM images with different surfactants including SDS, CTAB, F127, and PVP. (i) Atomic structures of $\mathrm{CoN}_{2+2}$ and $\mathrm{CoN}_{4}$ active sites of the Co-N-C catalysts. (j) Calculated free energy evolution diagram for the 4e ORR pathway on the $\mathrm{CoN}_{2+2}$ site under a limiting electrode potential of $U=0.73 \mathrm{~V}$ and on the $\mathrm{CoN}_{4}$ site under a limiting electrode potential of $U=0.67 \mathrm{~V}$. (k) Potential cycling $(0.6-1.0 \mathrm{~V})$ stability test of best Co-N-C@F127 catalysts in $\mathrm{O}_{2}-$ saturated $0.5 \mathrm{M} \mathrm{H}_{2} \mathrm{SO}_{4}$. Reproduced with permission from ref. 86. Copyright 2019, Royal Society of Chemistry. 
to contain substantial $\mathrm{CoN}_{2+2}$ sites, which are active and thermodynamically favorable for the four-electron ORR pathway (Fig. 8i and j). The Co-N-C@F127 catalyst exhibits an unprecedented ORR activity with a half-wave potential $\left(E_{1 / 2}\right)$ of $0.84 \mathrm{~V}(v s$. RHE) as well as enhanced stability in the corrosive acidic media (Fig. 8k). In a couple of recent studies, $\mathrm{N}$-doped carbon nanomaterials were selected as the supporting matrix for metal single atom catalysts to develop optimal $\mathrm{M}-\mathrm{N}_{4}$ sites, which can be active sites for oxygen adsorption and subsequent $\mathrm{O}=\mathrm{O}$ bond breaking during the ORR.

As aforementioned, the coordination between metal and heteroatoms in the carbon matrix, in particular, the $\mathrm{Co} / \mathrm{Fe}-$ $\mathrm{N}_{x}-\mathrm{C}$ moieties, can effectively modify the local electronic structure and thereby optimize the intermediate adsorption, leading to the prominent synergistic effect for enhancing activity. ${ }^{66,87,88}$ Especially, Zhang's group have fabricated a Co/N/O tri-doped graphene mesh catalyst (denoted as NGM-Co) with atomically dispersed $\mathrm{Co}-\mathrm{N}_{x}-\mathrm{C}$ active sites via the direct carbonization of a powdery mixture of gelatinized amylopectin, melamine, cobalt nitrate with the in situ generated $\mathrm{Mg}(\mathrm{OH})_{2}$ nanoflakes, as shown in Fig. 9a. ${ }^{89}$ The flexible solid $\mathrm{Zn}$-air battery displayed good cycling durability, due to the synergistic effect of high density of active sites derived from $\mathrm{Co}-\mathrm{N}_{x}-\mathrm{C}$ moieties, nitrogendoping and topological defects. Unlike nitrogen-doped carbon, $\mathrm{g}^{-} \mathrm{C}_{3} \mathrm{~N}_{4}$ has a 2D structure and can offer a fixed stoichiometric ratio with ordered pore structures and more active sites. ${ }^{90}$ For example, Cao's group has proposed a surfactant-assisted approach to synthesize the single atom $\mathrm{Co}-\mathrm{N}-\mathrm{C}$ catalysts (denoted as $\mathrm{CoN}_{4} /$ NG) via the pyrolysis of $\mathrm{g}_{-} \mathrm{C}_{3} \mathrm{~N}_{4}$, Co salt and the polyoxyethylenepolyoxypropylene-polyoxyethylene (PEO-PPO-PEO, marked as F127) complex precursor (Fig. 9b). ${ }^{91}$ The as-prepared $\mathrm{CoN}_{4} / \mathrm{NG}$ exhibited superior ORR/OER activity with the potential difference, $\Delta E\left(E_{\mathrm{OER}}-E_{\mathrm{ORR}}\right)$ between the OER potential at $10 \mathrm{~mA} \mathrm{~cm}{ }^{-2}$ and the ORR potential at $3 \mathrm{~mA} \mathrm{~cm}{ }^{-2}$ of $0.74 \mathrm{~V}$ (versus RHE), which is attributed to the synergetic effect of highly active sites of $\mathrm{CoN}_{4}$ moieties and pyridine nitrogen. Except for well-defined porphyrinlike $\mathrm{M}-\mathrm{N}_{4}$ sites, recent studies have demonstrated that introducing extra metals or heteroatoms to form new coordination structures is an effective strategy to further enhance the catalytic performance of single metallic sites catalysts. For instance, Yuan et al. prepared carbon nanosheets embedded with nitrogen and phosphorus dual-coordinated iron active sites (denoted as $\mathrm{Fe}-\mathrm{N} / \mathrm{P}-\mathrm{C}$ ) via the polymerization of pyrrole in the presence of phytic acid and $\mathrm{FeCl}_{3}$, as displayed in Fig. 9c. ${ }^{92}$ Both experimental and theoretical results suggest that the $\mathrm{Fe}-\mathrm{N}_{3} \mathrm{P}$ active sites can be favourable for the adsorption/desorption of oxygen intermediates, which would accelerate reaction kinetics and boost catalytic ORR performance.

\section{Novel air electrode development}

\subsection{Flexible free-standing 3D electrodes}

In terms of air electrodes applied in $\mathrm{Zn}$-air batteries, the current regular procedure is to combine electrocatalysts with polymer binders and then disperse them on the conductive substrate, such as carbon cloth (CC), carbon paper, stainless steel meshes, and metal foams. ${ }^{89,93-95}$ However, the resulting problem is that the exposed active sites of the catalysts are limited, and the synthetic processes are tedious. Thus, rechargeable flexible free-standing electrodes for $\mathrm{Zn}$-air batteries have attracted much attention as promising next-generation energy storage devices for portable and wearable electronics. ${ }^{96}$ Among other conductive substrates, CC is commonly utilized as a conductive substrate for air cathodes owing to its excellent flexibility, high conductivity, and stability. ${ }^{89}$ However, CC exhibits almost no catalytic activity and merely acts as a conductor of electricity. An ORR/OER bifunctional catalyst coated on a CC effectively alleviates the high overpotential of $\mathrm{Zn}$-air batteries. Experiments suggest that activating CCs by rational surface modification is a feasible, promising strategy to achieve activated carbon fibers used as bifunctional freestanding, flexible air electrodes without any binder. ${ }^{97}$ The design principles of carbon materials also apply to the surface modification of CCs, such as heteroatom doping, defect introduction, and in situ growth of other active components. For example, Zhang's group proposed the exfoliation of carbon fibers using the high-temperature $\mathrm{H}_{2}$ etching method, with which an emerging core-shell structure of carbon fibers coated by active porous graphene nanosheet skin can be obtained. ${ }^{97}$ The core is an internal free-standing flexible, conductive carbon fiber skeleton, while the outer carbon shell consists of porous graphene sheets through directly exfoliating on the surface. The coaxial cable-like structure $o-\mathrm{CC}-\mathrm{H}_{2}$ with abundant defects and a porous surface exhibits a high electrocatalytic activity and is assembled as the air electrode in a flexible solid-state $\mathrm{Zn}$-air battery with an open-circuit voltage of $1.258 \mathrm{~V}$. Zhao's group presented a chemical oxidation CC by mild acid oxidation followed by air calcination methods (Fig. 10a) ${ }^{98}$ Uniform nanoporous, super hydrophilic surface structures with optimized oxygen-rich functional groups and an enhanced surface area can be achieved in this manner. Two-step-activated CC (denoted as CC-AC) as a metal-free OER electrocatalyst exhibits an overpotential of $360 \mathrm{mV}$ at a current density of $10 \mathrm{~mA} \mathrm{~cm}{ }^{-2}$ in $1 \mathrm{M} \mathrm{KOH}$. When applied as a binder-free air cathode in a rechargeable flexible $\mathrm{Zn}$-air battery, a peak power density of $52.3 \mathrm{~mW} \mathrm{~cm}^{-2}$ and a good flexibility are achieved (Fig. 10b). In recent years, CC composites with embedded metal/metal oxides have become widespread by altering geometric structures and compositions..$^{99-101}$ Manganese oxide is an attractive candidate of $\mathrm{Zn}$-air batteries due to the advantages of abundant resources, low cost, various valence states, and multiple crystal structures. ${ }^{25}$ However, its poor electrical conductivity hinders the development of the ORR and OER bifunctional electrocatalytic catalysts. Zhang's group reported an in situ growth of $\mathrm{MnO}_{x}$ nanoplates on CC via a chemical bath deposition followed by a thermally driven phase transformation strategy to address this issue (Fig. 10c). ${ }^{102}$ Redox reactions between carbon and manganese oxide generated the porous surface of the carbon fiber and facilitated the crystal structure transition of manganese oxide. Furthermore, galvanostatic discharge-charge cycling curves with different bending angles were obtained to investigate the flexibility. Fig. 10d illustrates that the flexible solid state $\mathrm{Zn}$-air batteries can steadily operate for at least $30 \mathrm{~h}$ with bending angles at $60^{\circ}$, 


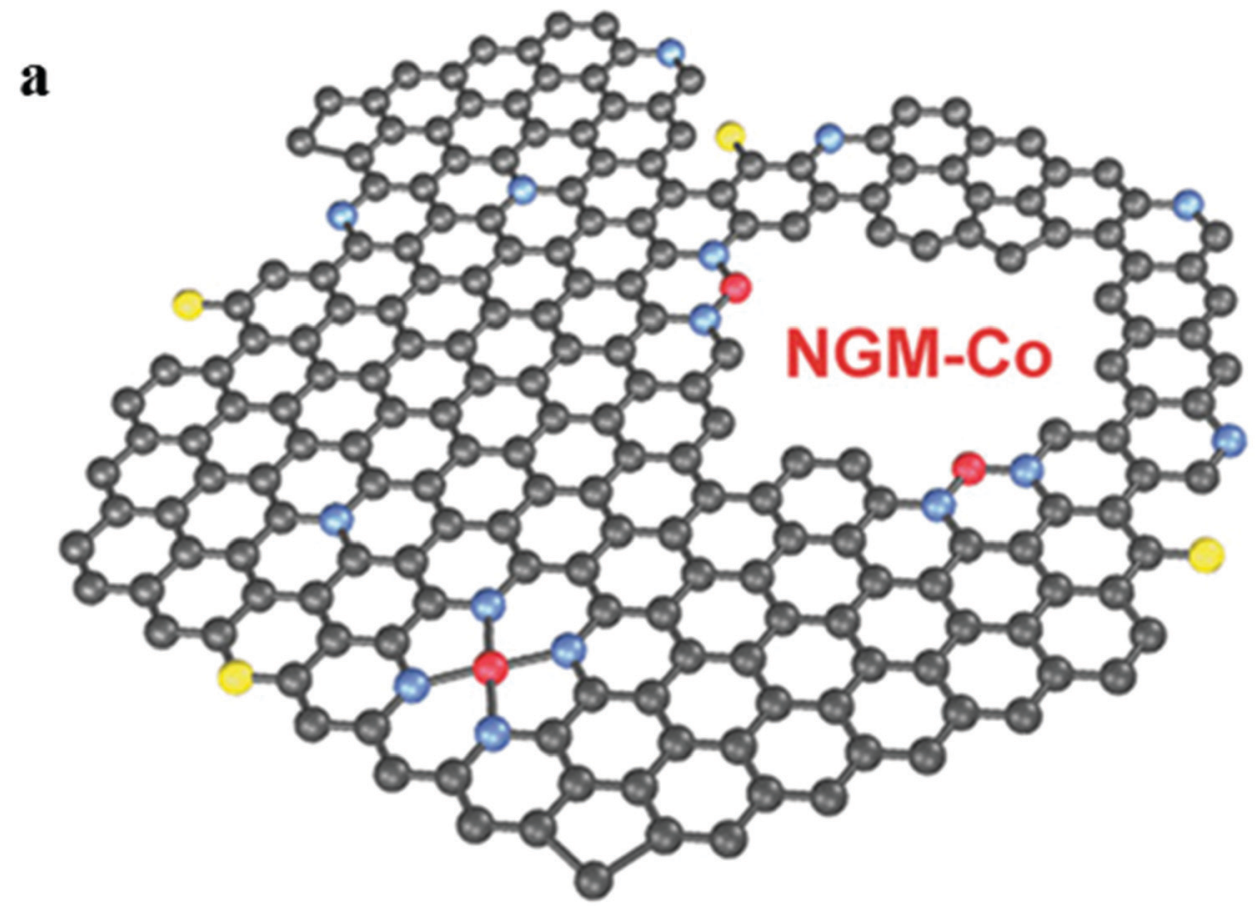

b

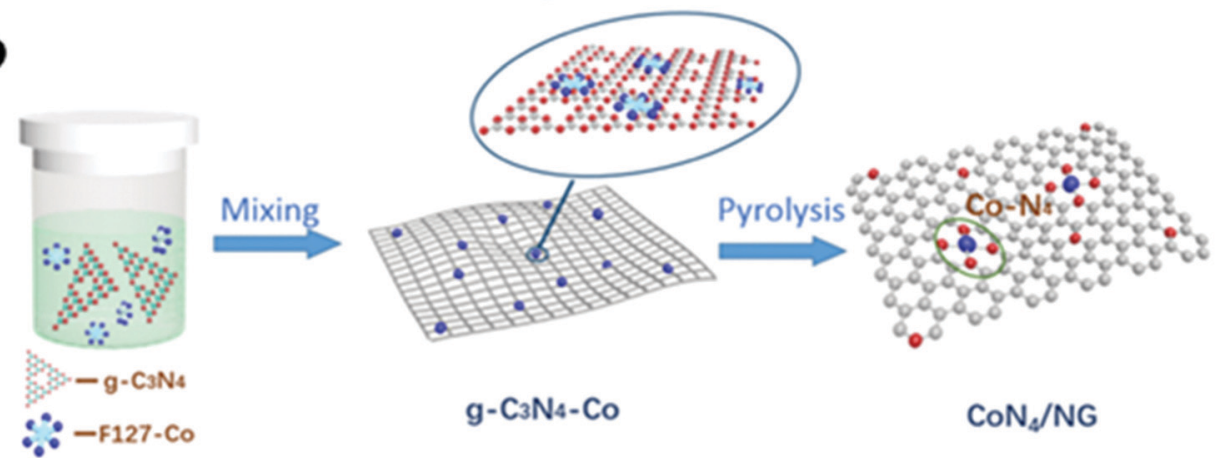

c

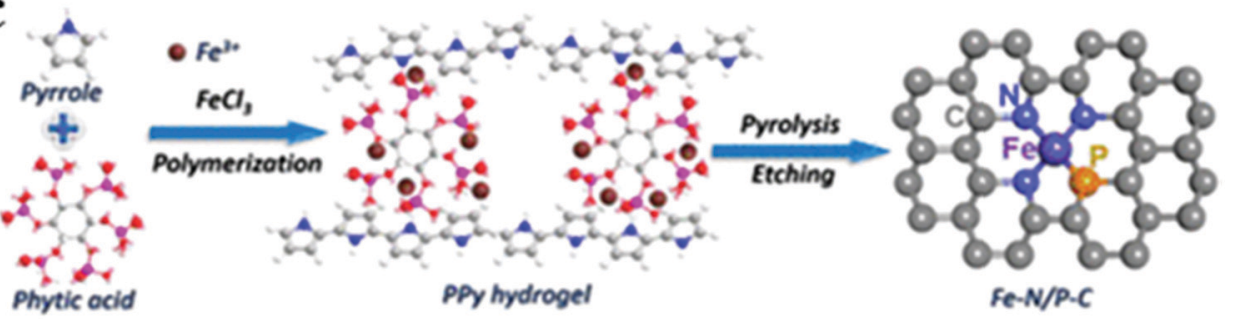

Fig. 9 Schematic illustration of the synthesis of atomically dispersed $\mathrm{M}-\mathrm{N}-\mathrm{C}$ sites in traditional carbon-based materials. (a) Hierarchical Co/N/O tridoped graphene catalyst (NGM-Co). Reproduced with permission from ref. 89. Copyright 2017, Wiley-VCH. (b) Single atom Co-N-C catalysts (CoN 4 / NG). Reproduced with permission from ref. 91. Copyright 2018, Elsevier. (c) Nitrogen and phosphorus dual-coordinated iron active sites (Fe-N/P-C). Reproduced with permission from ref. 92. Copyright 2019, American Chemical Society.

$90^{\circ}, 120^{\circ}$, and $180^{\circ}$. The compact interaction between the electrode and the polymer gel electrolyte would improve the cycling life of the battery as the bending angles increase. Recently, electro spun carbon nanofibers from polyacrylonitrile (PAN) have been considered appropriate flexible 3D scaffolds for constructing superior composite electrocatalysts owing to their high electronic conductivity, high inter-fiber porosity, excellent chemical stability, high-temperature resistance, and large surface area. ${ }^{103}$ For example, Yan's group utilized the CoFe-glycerate microspheres as a metal source as well as a hard template to synthesize the tunable PAN-derived porous electro spun carbon nanofiber catalysts (Fig. 10e). ${ }^{104}$ These carbon fibers exhibited good ORR and OER bifunctional catalytic performance, which might be attributed to the features of abundant dispersed active $\mathrm{M}-\mathrm{N}-\mathrm{C}$ sites, hydrophilicity, and porous structures, thus leading to an efficient mass transfer and available solid-liquid-gas reaction interfaces. Fig. 10f shows that the assembled flexible rechargeable $\mathrm{Zn}$-air batteries using a $\mathrm{KOH}$-filled alkaline polyacrylate hydrogel 


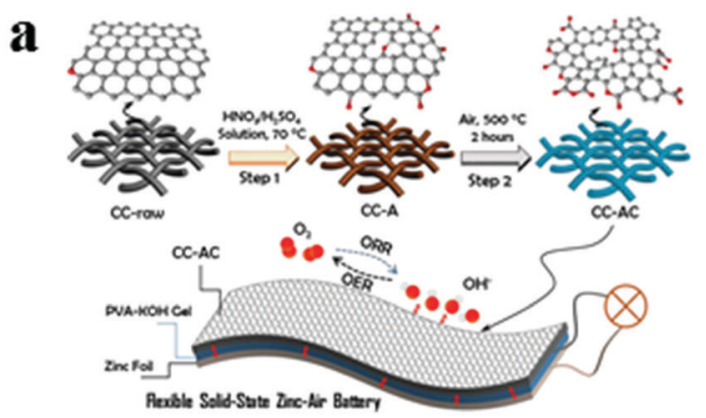

C

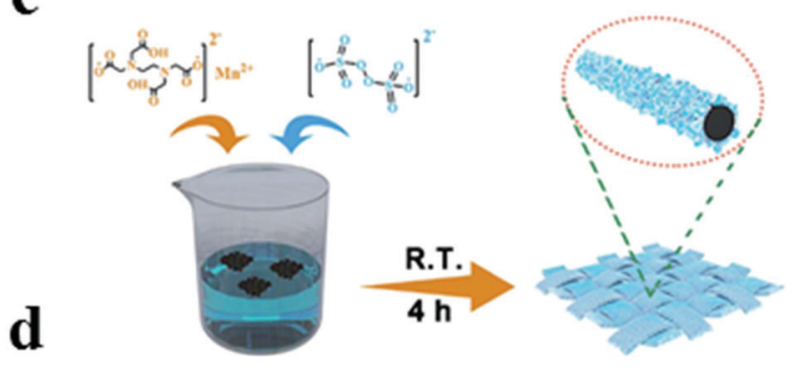

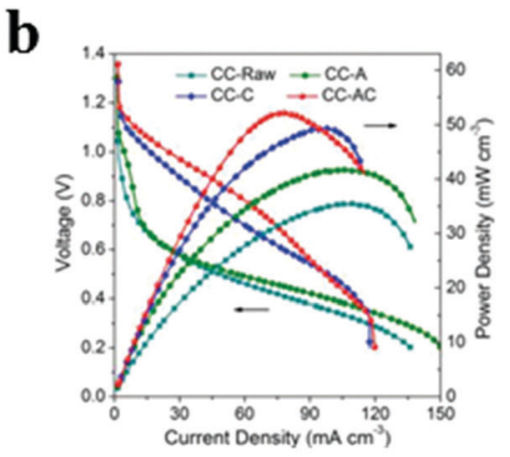

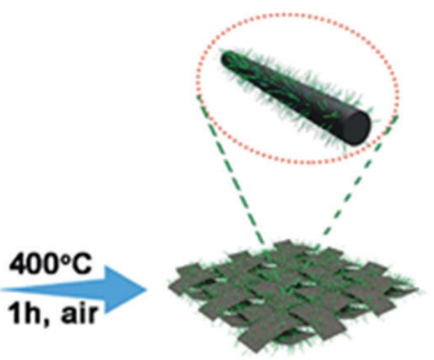

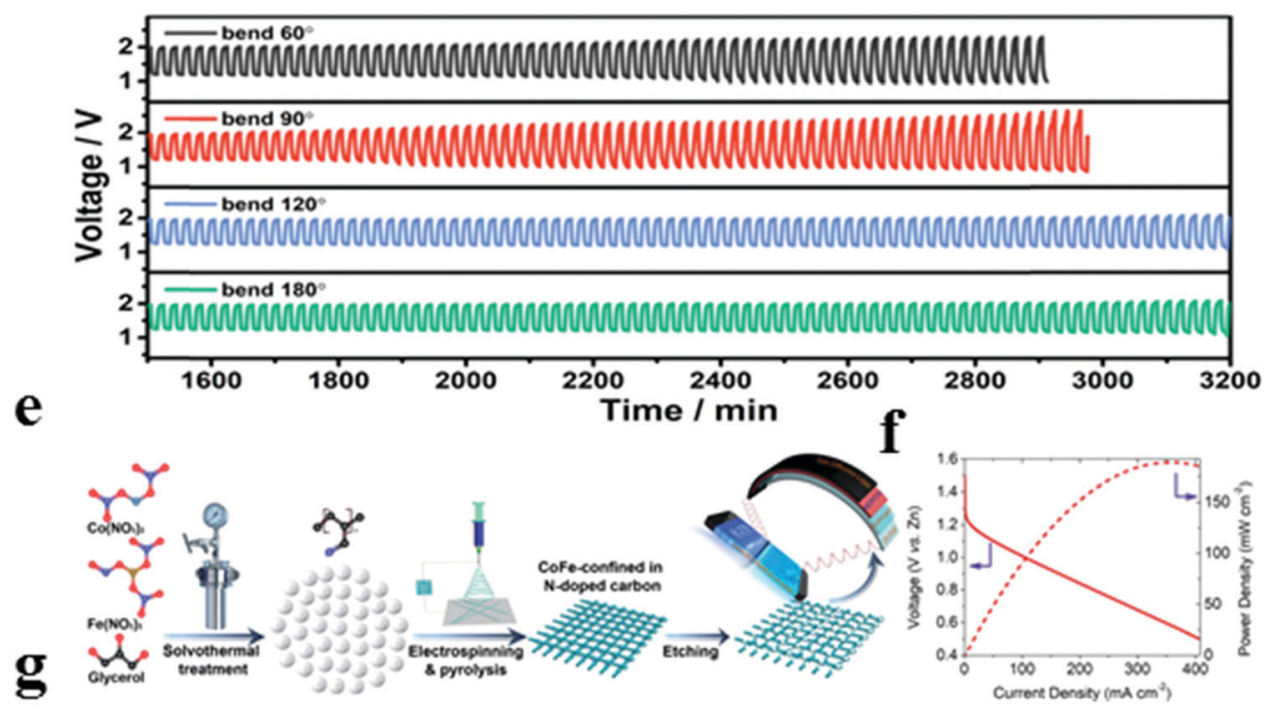

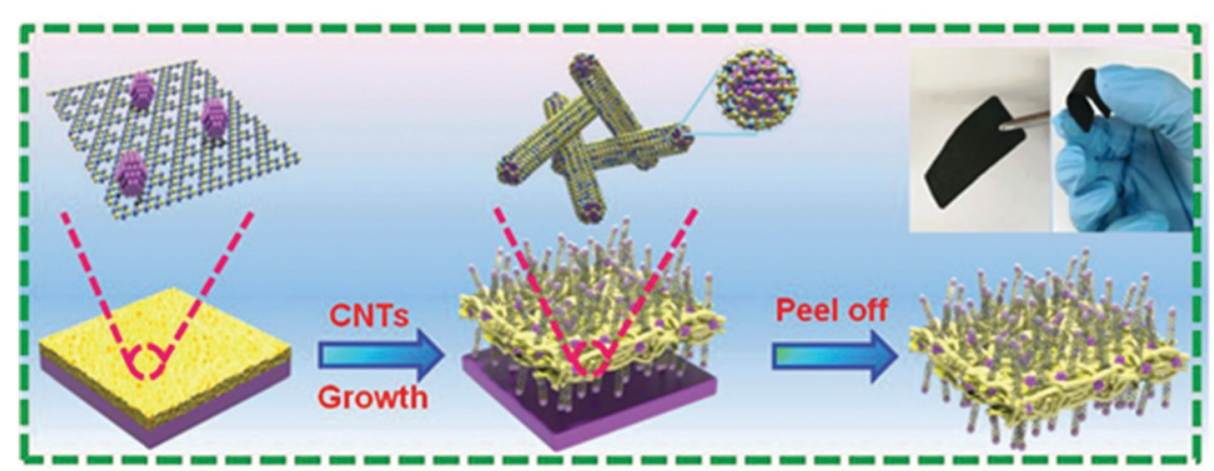

Fig. 10 (a) Schematic illustration of the two-step activation method for the fabrication of CC-AC. (b) Polarization and power density curves of flexible $\mathrm{Zn}$-air batteries using CC-AC, CC-C, CC-A, and CC-raw as the air cathode. Reproduced with permission from ref. 98. Copyright 2018, Wiley-VCH. (c) Schematic illustration of the synthesis of $\mathrm{MnO}_{x}-\mathrm{CC}-400$. (d) Stability test of the flexible $\mathrm{Zn}$-air batteries under different bending angles. Reproduced with permission from ref. 102. Copyright 2019, Royal Society of Chemistry. (e) Schematic illustration of the preparation of metal-doped porous carbon nanofibers. (f) Power density and discharge profiles for the MDPCF-based solid-state flexible ZAB. Reproduced with permission from ref. 104. Copyright 2020, Royal Society of Chemistry. (g) Formation mechanism of Co/CNFs. Reproduced with permission from ref. 105. Copyright 2019, Wiley-VCH. 
electrolyte exhibited a peak power density of $188.6 \mathrm{~mW} \mathrm{~cm}^{-2}$ at $371.3 \mathrm{~mA} \mathrm{~cm}^{-2}$. In addition, Wu's group demonstrated a novel, self-supporting, binder-free film electrode, which could simultaneously catalyze the ORR, OER, and HER with excellent activities and superior stability. ${ }^{105}$ Fig. $10 \mathrm{~g}$ illustrates the fabrication of cobalt NP-encapsulated 3D conductive films (denoted as Co/CNFs). First, melamine is converted into nitrogen carbon on the Co foil at a low pyrolysis temperature. Volatile Co species can be generated from the Co foil and are trapped by the $\mathrm{N}$-doped carbon layer with the increase of carbonization temperature. The Co species can aggregate into Co NPs and as seeds for the self-catalytic growth of N-doped carbon nanotubes, forming the 3D assembly of Co NP embedded carbon nanotube films. This solid diffusion strategy provides a new, feasible method to fabricate an "all in one" free-standing multifunctional 3D electrode.

\subsection{Air electrodes with new concept $\mathrm{Zn}$-air batteries}

Conventional alkaline $\mathrm{Zn}$-air batteries have attracted extensive attention and research. However, the highly alkaline reaction environment has a few disadvantages, such as strong corrosivity and easily being subjected to carbon dioxide in the air and zinc dendrites. In recent research, rechargeable $\mathrm{Zn}$-air batteries with neutral aqueous electrolytes were proposed, which could alleviate the issue of failure of electrolytes and formation of zinc dendrites during the charge and discharge cycles. ${ }^{106,107}$ However, the lower ionic conductivity of neutral electrolytes and the low efficiency of ORR and OER catalysts substantially hampered the development of neutral $\mathrm{Zn}$-air batteries. Engineering rational heterostructures within catalysts is an efficient method to improve the catalytic properties via interfacial electronic coupling. For example, Zhang's group reported $\mathrm{NiFe}_{2} \mathrm{O}_{4} / \mathrm{FeNi}_{2} \mathrm{~S}_{4}$ heterostructured nanosheets (denoted as $\mathrm{NiFe}_{2} \mathrm{O}_{4} / \mathrm{FeNi}_{2} \mathrm{~S}_{4} \mathrm{HNSs}$ ) supported on carbon fiber paper via a wet chemical sulfidation method. ${ }^{108}$ The heterostructure interface effect in electrocatalysis can be regulated by employing different sulfidation times. The $\mathrm{FeNi}_{2} \mathrm{~S}_{4}$ ratios increased with the increase of the reaction time, suggesting that the controlled sulfidation is a feasible strategy to tune the oxide/sulfide interface intensities and profiles, thus obtaining an ideal bifunctional oxygen performance. Alkaline Zn-air batteries with $6 \mathrm{M} \mathrm{KOH}$ and neutral $\mathrm{Zn}$-air batteries with $4.0 \mathrm{M} \mathrm{NH}_{4} \mathrm{Cl}$ and $2.0 \mathrm{M} \mathrm{KCl}$ $(\mathrm{pH}=7.0)$ were constructed to demonstrate the advantages of using a neutral electrolyte. $\mathrm{NiFe}_{2} \mathrm{O}_{4} / \mathrm{FeNi}_{2} \mathrm{~S}_{4}$ HNS neutral $\mathrm{Zn}$-air batteries can steadily run for 900 cycles at a current density of $0.5 \mathrm{~mA} \mathrm{~cm} \mathrm{~cm}^{-2}$ with a roundtrip efficiency of only $0.6 \%$ decay, suggesting the superior cycling stability for low current devices. With the increase of emerging flexible devices, Chen's group prepared a bamboo-structured 1D fibrous electrocatalyst and a freezing-tolerant hydrogel electrolyte, which exhibited excellent low-temperature adaptability for flexible rechargeable $\mathrm{Zn}$-air batteries. ${ }^{109}$ The high anti-freezing properties of gel electrolytes are closely correlated with the strong polarity of terminal groups. Coaxial electrospinning can introduce abundant porosity and maximize the accessibility of active sites inside the conductive fibers. More recently, Li's group demonstrated a photo-excited Zn-air battery with two cathodes of poly(1,4-di(2-thienyl))benzene
(PDTB) and $\mathrm{TiO}_{2}$ grown on carbon papers to sandwich a $\mathrm{Zn}$ anode. ${ }^{110}$ The PDTB and $\mathrm{TiO}_{2}$ cathode can capture light to separate electrons and holes, thus facilitating the ORR and OER, respectively. In the discharging process, the photoelectrons in the conduction bands of PDTB can reduce $\mathrm{O}_{2}$ to $\mathrm{O}_{2}{ }^{{ }^{-}}$, and $\mathrm{O}_{2}{ }^{*-}$ then converts to $\mathrm{OH}^{-}$. In addition, the holes left behind in the valence bands can oxidize $\mathrm{Zn}$ to $\mathrm{ZnO}$. In the charging process on $\mathrm{TiO}_{2}$, the holes driven by an applied voltage can oxidize $\mathrm{OH}^{-}$ to $\mathrm{OH}^{*}$ and then decomposes to release $\mathrm{O}_{2}$. The discharge voltage of the photo-excited $\mathrm{Zn}$-air battery is as high as $1.90 \mathrm{~V}$, and the charge voltage of that is $0.59 \mathrm{~V}$ owing to the additional light contribution. These studies provide an innovative pathway for designing and developing efficient rechargeable $\mathrm{Zn}$-air batteries as next generation energy storage devices.

\section{Conclusions and outlook}

Carbon-based nanomaterials have been regarded as one of the most promising catalysts for electrocatalysis and energy conversion technologies. Specially, bifunctional ORR/OER catalysts with good electrical conductivity, competitive electrocatalytic activity and relatively high chemical stability are critical for exploring efficient rechargeable $\mathrm{Zn}$-air batteries. In the last five years, considerable effort has been devoted to developing, designing, and synthesizing cost effective, and high-efficiency bifunctional electrocatalysts, such as carbon-based nanomaterials and transition metals/alloys, metal oxides, nitrides, carbides, phosphides and chalcogenides. Rational integration of multiple active components has been acknowledged as a feasible way to increase the electrocatalytic performance. This mini-review sheds light on research hotspot advances of carbon-based bifunctional oxygen catalysts employed as air-cathodes for $\mathrm{Zn}$-air batteries and discusses carbon-supported non-precious metal-based materials, which involve heteroatom-doped carbon loading metal-based species and metal organic framework-derived $\mathrm{M}-\mathrm{N}-\mathrm{C}(\mathrm{M}=\mathrm{Fe}$, $\mathrm{Co}, \mathrm{Ni}, \mathrm{Cu}$ ) composites. Heteroatom-doping, defect engineering and hybridization are effective strategies to tune the interface defects and electronic structure, and thus enhance the catalytic performance. Moreover, the catalytic activity and stability would be improved synergistically by designing suitable components, optimizing structures/morphologies, and constructing a unique synergistic effect between the active components and conductive carbon. The dispersion of active sites and regulation of physicochemical properties including crystalline phases and transition metal valence are also of great importance. Although significant progress has been made, several challenges remain in the development of desired carbon-based bifunctional catalysts: (1) developing in situ technologies. A well-defined active site is a vital factor for the electrocatalytic activity of catalysts. As previously reported, the phase and morphology of the catalyst may change qualitatively especially during the OER reaction process. Consequently, it is significantly crucial to track the species and structure of active sites via in situ characterization technologies combined with the experimental observation. In addition, combining in situ techniques with DFT calculations is favorable to 
understand the ORR/OER reaction mechanisms, and even might deepen the understanding of the reaction mechanisms in carbonbased catalysts assembled in Zn-air batteries. (2) Optimizing synthesis methods. The current carbon-based materials generally rely on template methods, hydrothermal and multiple high temperature pyrolysis. However, these methods possess some disadvantages, such as sophisticated synthetic procedures and extremely low yields. Furthermore, it is merely applicable to certain catalysts. Thus, feasible and universal synthetic approaches should to be developed. (3) Exploring corrosion-resistant carbon-based materials. Carbon corrosion leads to the poor catalytic activity and stability of bifunctional catalysts, which would peel off or aggravate the aggregation of active species. To accelerate practical electrocatalytic applications, new corrosion-resistant carbon-based materials should be developed. (4) Developing novel 3D freestanding cathodes. Active components strongly anchoring to a stable conducting substrate might be an effective strategy to prepare highly efficient, stable catalysts. The 3D porous freestanding carbon electrodes can expose more active sites and offer infinite possibilities for the development of flexible $\mathrm{Zn}$-air batteries. Therefore, despite the important breakthroughs in the development of carbon-based catalysts, challenges remain in applying these to practical $\mathrm{Zn}$-air batteries. This review offers an update on the recent advances of carbon-based bifunctional oxygen catalysts. We hope that this will provide several helpful insights into the future development of carbon-based eletrocatalysts.

\section{Conflicts of interest}

There are no conflicts to declare.

\section{Notes and references}

1 Y. Li and H. Dai, Chem. Soc. Rev., 2014, 43, 5257-5275.

2 X. Chen, Z. Zhou, H. E. Karahan, Q. Shao, L. Wei and Y. Chen, Small, 2018, 14, e1801929.

3 D. Yang, L. Zhang, X. Yan and X. Yao, Small Methods, 2017, 1, 1700209.

4 Y. Jiao, Y. Zheng, M. Jaroniec and S. Z. Qiao, J. Am. Chem. Soc., 2014, 136, 4394-4403.

5 Y. Zheng, Y. Jiao, M. Jaroniec, Y. Jin and S. Z. Qiao, Small, 2012, 8, 3550-3566.

6 B. Li, Y. Chen, X. Ge, J. Chai, X. Zhang, T. S. Hor, G. Du, Z. Liu, H. Zhang and Y. Zong, Nanoscale, 2016, 8, 5067-5075.

7 X. Wu, G. Meng, W. Liu, T. Li, Q. Yang, X. Sun and J. Liu, Nano Res., 2017, 11, 163-173.

8 Q. Wang, L. Shang, R. Shi, X. Zhang, Y. Zhao, G. I. N. Waterhouse, L.-Z. Wu, C.-H. Tung and T. Zhang, Adv. Energy Mater., 2017, 7, 1700467.

9 F. Meng, H. Zhong, D. Bao, J. Yan and X. Zhang, J. Am. Chem. Soc., 2016, 138, 10226-10231.

10 J. Ortiz-Medina, Z. Wang, R. Cruz-Silva, A. Morelos-Gomez, F. Wang, X. Yao, M. Terrones and M. Endo, Adv. Mater., 2019, 31, e1805717.

11 D. Liu, L. Dai, X. Lin, J. F. Chen, J. Zhang, X. Feng, K. Mullen, X. Zhu and S. Dai, Adv. Mater., 2019, 31, e1804863.
12 X. Wang, A. Vasileff, Y. Jiao, Y. Zheng and S. Z. Qiao, Adv. Mater., 2019, 31, e1803625.

13 S. Zhao, D. W. Wang, R. Amal and L. Dai, Adv. Mater., 2019, 31, e1801526.

14 Q. Zhao, Z. Yan, C. Chen and J. Chen, Chem. Rev., 2017, 117, 10121-10211.

15 Y. Guo, Y.-N. Chen, H. Cui and Z. Zhou, Chin. J. Catal., 2019, 40, 1298-1310.

16 L. Yang, J. Shui, L. Du, Y. Shao, J. Liu, L. Dai and Z. Hu, Adv. Mater., 2019, 31, e1804799.

17 L. Zhang, C. Y. Lin, D. Zhang, L. Gong, Y. Zhu, Z. Zhao, Q. Xu, H. Li and Z. Xia, Adv. Mater., 2019, 31, e1805252.

18 W. X. Justus Masa, M. Muhler and W. Schuhmann, Angew. Chem., Int. Ed., 2015, 54, 10102-10120.

19 Y. Jiao, Y. Zheng, K. Davey and S.-Z. Qiao, Nat. Energy, 2016, 1, 16130.

20 J. Zhang, H. Li, P. Guo, H. Ma and X. S. Zhao, J. Mater. Chem. A, 2016, 4, 8497-8511.

21 J. Zhang and L. Dai, Angew. Chem., Int. Ed., 2016, 55, 13296-13300.

22 J. Zhang and L. Dai, ACS Catal., 2015, 5, 7244-7253.

23 L. Shang, H. Yu, X. Huang, T. Bian, R. Shi, Y. Zhao, G. I. Waterhouse, L. Z. Wu, C. H. Tung and T. Zhang, Adv. Mater., 2016, 28, 1668-1674.

24 J. Zhang, Z. Zhao, Z. Xia and L. Dai, Nat. Nanotechnol., 2015, 10, 444-452.

25 S. Chen, L. Zhao, J. Ma, Y. Wang, L. Dai and J. Zhang, Nano Energy, 2019, 60, 536-544.

26 Z. Chen, Q. Wang, X. Zhang, Y. Lei, W. Hu, Y. Luo and Y. Wang, Sci. Bull., 2018, 63, 548-555.

27 S. Wang, H. Jiang and L. Song, Batteries Supercaps, 2019, 2, 509-523.

28 W. Li, D. Wang, Y. Zhang, T. Li, T. Wang, Y. Zou, Y. Wang, R. Chen and S. Wang, Adv. Mater., 2020, 32, e1907879.

29 X. Yan, Y. Jia and X. Yao, Chem. Soc. Rev., 2018, 47, 7628-7658.

30 Y. Yin, X. Sun, M. Zhou, X. Zhao, J. Qin, S. Z. Qiao, X. W. Du and J. Yang, ChemCatChem, 2019, 11, 6131-6138.

31 Q. Wang, Y. Lei, D. Wang and Y. Li, Energy Environ. Sci., 2019, 12, 1730.

32 Y. Zhang, L. Tao, C. Xie, D. Wang, Y. Zou, R. Chen, Y. Wang, C. Jia and S. Wang, Adv. Mater., 2020, 32, 1905923.

33 J. Zhu, Y. Huang, W. Mei, C. Zhao, C. Zhang, J. Zhang, I. S. Amiinu and S. Mu, Angew. Chem., Int. Ed., 2019, 58, 3859-3864.

34 Y. Jia, L. Zhang, A. Du, G. Gao, J. Chen, X. Yan, C. L. Brown and X. Yao, Adv. Mater., 2016, 28, 9532-9538.

35 Y. Guo, X. Gao, C. Zhang, Y. Wu, X. Chang, T. Wang, X. Zheng, A. Du, B. Wang, J. Zheng, K. Ostrikov and X. Li, J. Mater. Chem. A, 2019, 7, 8129-8135.

36 Z. Wang, Y. Zhang, E. C. Neyts, X. Cao, X. Zhang, B. W. L. Jang and C.-j. Liu, ACS Catal., 2018, 8, 2093-2110.

37 L. Xu, Q. Jiang, Z. Xiao, X. Li, J. Huo, S. Wang and L. Dai, Angew. Chem., Int. Ed., 2016, 55, 5277-5281.

38 S. Chen, Z. Chen, S. Siahrostami, T. R. Kim, D. Nordlund, D. Sokaras, S. Nowak, J. W. F. To, D. Higgins, R. Sinclair, 
J. K. Nørskov, T. F. Jaramillo and Z. Bao, ACS Sustainable Chem. Eng., 2017, 6, 311-317.

39 X. Shu, S. Chen, S. Chen, W. Pan and J. Zhang, Carbon, 2020, 157, 234-243.

40 S. Chen, S. Chen, B. Zhang and J. Zhang, ACS Appl. Mater. Interfaces, 2019, 11, 16720-16728.

41 B. Ni, C. Ouyang, X. Xu, J. Zhuang and X. Wang, Adv. Mater., 2017, 29, 1701354.

42 L. Yang, D. Wang, Y. Lv and D. Cao, Carbon, 2019, 144, 8-14.

43 D. Chen, J. Zhu, X. Mu, R. Cheng, W. Li, S. Liu, Z. Pu, C. Lin and S. Mu, Appl. Catal., B, 2020, 268, 118729.

44 S. Gao, B. Fan, R. Feng, C. Ye, X. Wei, J. Liu and X. Bu, Nano Energy, 2017, 40, 462-470.

45 C. C. Yang, S. F. Zai, Y. T. Zhou, L. Du and Q. Jiang, Adv. Funct. Mater., 2019, 1901949.

46 A. Parra-Puerto, K. L. Ng, K. Fahy, A. E. Goode, M. P. Ryan and A. Kucernak, ACS Catal., 2019, 9, 11515-11529.

47 Y. Lin, L. Yang, Y. Zhang, H. Jiang, Z. Xiao, C. Wu, G. Zhang, J. Jiang and L. Song, Adv. Energy Mater., 2018, 8, 1703623.

48 D. M. Morales, M. A. Kazakova, S. Dieckhöfer, A. G. Selyutin, G. V. Golubtsov, W. Schuhmann and J. Masa, Adv. Funct. Mater., 2019, 30, 1905992.

49 W. Cheng, P. Yuan, Z. Lv, Y. Guo, Y. Qiao, X. Xue, X. Liu, W. Bai, K. Wang, Q. Xu and J. Zhang, Appl. Catal., B, 2020, 260, 118198.

50 X. Hu, Y. Min, L.-L. Ma, J.-Y. Lu, H.-C. Li, W.-J. Liu, J.-J. Chen and H.-Q. Yu, Appl. Catal., B, 2020, 268, 118405.

51 X. Sun, S. Sun, S. Gu, Z. Liang, J. Zhang, Y. Yang, Z. Deng, P. Wei, J. Peng, Y. Xu, C. Fang, Q. Li, J. Han, Z. Jiang and Y. Huang, Nano Energy, 2019, 61, 245-250.

52 Y. Han, Y. G. Wang, W. Chen, R. Xu, L. Zheng, J. Zhang, J. Luo, R. A. Shen, Y. Zhu, W. C. Cheong, C. Chen, Q. Peng, D. Wang and Y. Li, J. Am. Chem. Soc., 2017, 139, 17269-17272.

53 P. Yin, T. Yao, Y. Wu, L. Zheng, Y. Lin, W. Liu, H. Ju, J. Zhu, X. Hong, Z. Deng, G. Zhou, S. Wei and Y. Li, Angew. Chem., Int. Ed., 2016, 55, 10800-10805.

54 P. Yu, L. Wang, F. Sun, Y. Xie, X. Liu, J. Ma, X. Wang, C. Tian, J. Li and H. Fu, Adv. Mater., 2019, 1901666.

55 Y. Lian, W. Yang, C. Zhang, H. Sun, Z. Deng, W. Xu, L. Song, Z. Ouyang, Z. Wang, J. Guo and Y. Peng, Angew. Chem., Int. Ed., 2020, 59, 286-294.

56 Y. Sun, J. Wang, Q. Liu, M. Xia, Y. Tang, Y. Hou, J. Tse and Y. Zhao, J. Mater. Chem. A, 2019, 7, 27175.

57 X. Zhao, X. Liu, B. Huang, P. Wang and Y. Pei, J. Mater. Chem. A, 2019, 7, 24583-24593.

58 Y. Mun, S. Lee, K. Kim, S. Kim, S. Lee, J. W. Han and J. Lee, J. Am. Chem. Soc., 2019, 141, 6254-6262.

59 J. Gu, S. Magagula, J. Zhao and Z. Chen, Small Methods, 2019, 3, 1800550.

60 H. F. Wang, L. Chen, H. Pang, S. Kaskel and Q. Xu, Chem. Soc. Rev., 2020, 49, 1414-1448.

61 J. Zhu, H. Zhou, C. Zhang, J. Zhang and S. Mu, Nanoscale, 2017, 9, 13257-13263.
62 X. Zheng, J. Wu, X. Cao, J. Abbott, C. Jin, H. Wang, P. Strasser, R. Yang, X. Chen and G. Wu, Appl. Catal., B, 2019, 241, 442-451.

63 X. Zhu, C. Hu, R. Amal, L. Dai and X. Lu, Energy Environ. Sci., 2020, DOI: 10.1039/d0ee02800b.

64 Y. Guo, S. Yao, L. Gao, A. Chen, M. Jiao, H. Cui and Z. Zhou, J. Mater. Chem. A, 2020, 8, 4386-4395.

65 Y. Li, Y. Tong and F. Peng, J. Energy Chem., 2020, 48, 308-321.

66 Y. N. Chen, X. Zhang and Z. Zhou, Small Methods, 2019, 3, 1900050.

67 Y. Zhu, Z. Zhang, Z. Lei, Y. Tan, W. Wu, S. Mu and N. Cheng, Carbon, 2020, 167, 188-195.

68 J. Zhu, M. Xiao, Y. Zhang, Z. Jin, Z. Peng, C. Liu, S. Chen, J. Ge and W. Xing, ACS Catal., 2016, 6, 6335-6342.

69 G. Huang, Z. Xiao, R. Chen and S. Wang, ACS Sustainable Chem. Eng., 2018, 6, 15954-15969.

70 Z. Xiao, Y. Wang, Y.-C. Huang, Z. Wei, C.-L. Dong, J. Ma, S. Shen, Y. Li and S. Wang, Energy Environ. Sci., 2017, 10, 2563-2569.

71 L. Wei, H. E. Karahan, S. Zhai, H. Liu, X. Chen, Z. Zhou, Y. Lei, Z. Liu and Y. Chen, Adv. Mater., 2017, 29, 1701410.

72 H. Luo, W. J. Jiang, S. Niu, X. Zhang, Y. Zhang, L. P. Yuan, C. He and J. S. Hu, Small, 2020, 16, e2001171.

73 J. Fu, F. M. Hassan, C. Zhong, J. Lu, H. Liu, A. Yu and Z. Chen, Adv. Mater., 2017, 29, 1702526.

74 C. Lai, M. Gong, Y. Zhou, J. Fang, L. Huang, Z. Deng, X. Liu, T. Zhao, R. Lin, K. Wang, K. Jiang, H. Xin and D. Wang, Appl. Catal., B, 2020, 274, 119086.

75 G. Zhang, Y. Jia, C. Zhang, X. Xiong, K. Sun, R. Chen, W. Chen, Y. Kuang, L. Zheng, H. Tang, W. Liu, J. Liu, X. Sun, W.-F. Lin and H. Dai, Energy Environ. Sci., 2019, 12, 1317-1325.

76 B. Zhang, H. Wang, Z. Zuo, H. Wang and J. Zhang, J. Mater. Chem. A, 2018, 6, 15728-15737.

77 Q. Zhou, Z. Zhang, J. Cai, B. Liu, Y. Zhang, X. Gong, X. Sui, A. Yu, L. Zhao, Z. Wang and Z. Chen, Nano Energy, 2020, 71, 104592.

78 W. Zang, A. Sumboja, Y. Ma, H. Zhang, Y. Wu, S. Wu, H. Wu, Z. Liu, C. Guan, J. Wang and S. J. Pennycook, ACS Catal., 2018, 8, 8961-8969.

79 Q. Xu, H. Jiang, Y. Li, D. Liang, Y. Hu and C. Li, Appl. Catal., B, 2019, 256, 117893.

80 Y. Chen, S. Ji, Y. Wang, J. Dong, W. Chen, Z. Li, R. Shen, L. Zheng, Z. Zhuang, D. Wang and Y. Li, Angew. Chem., Int. Ed., 2017, 56, 6937-6941.

81 D. Ding, K. Shen, X. Chen, H. Chen, J. Chen, T. Fan, R. Wu and Y. Li, ACS Catal., 2018, 8, 7879-7888.

82 N. K. Wagh, S. S. Shinde, C. H. Lee, J.-Y. Jung, D.-H. Kim, S.-H. Kim, C. Lin, S. U. Lee and J.-H. Lee, Appl. Catal., B, 2020, 268, 118746.

83 Y. Lin, P. Liu, E. Velasco, G. Yao, Z. Tian, L. Zhang and L. Chen, Adv. Mater., 2019, 31, e1808193.

84 H. Yang and X. Wang, Adv. Mater., 2019, 31, e1800743.

85 X. Han, X. Ling, D. Yu, D. Xie, L. Li, S. Peng, C. Zhong, N. Zhao, Y. Deng and W. Hu, Adv. Mater., 2019, 31, e1905622. 
86 Y. He, S. Hwang, D. A. Cullen, M. A. Uddin, L. Langhorst, B. Li, S. Karakalos, A. J. Kropf, E. C. Wegener, J. Sokolowski, M. Chen, D. Myers, D. Su, K. L. More, G. Wang, S. Litster and G. Wu, Energy Environ. Sci., 2019, 12, 250-260.

87 H. Liu, X. Liu, W. Li, X. Guo, Y. Wang, G. Wang and D. Zhao, Adv. Energy Mater., 2017, 7, 1700283.

88 J. Guo, J. Huo, Y. Liu, W. Wu, Y. Wang, M. Wu, H. Liu and G. Wang, Small Methods, 2019, 3, 1900159.

89 C. Tang, B. Wang, H. F. Wang and Q. Zhang, Adv. Mater., 2017, 29, 1703185.

90 S. M. Tan and M. Pumera, ACS Nano, 2019, 13, 2681-2728.

91 L. Yang, L. Shi, D. Wang, Y. Lv and D. Cao, Nano Energy, 2018, 50, 691-698.

92 K. Yuan, D. Lutzenkirchen-Hecht, L. Li, L. Shuai, Y. Li, R. Cao, M. Qiu, X. Zhuang, M. K. H. Leung, Y. Chen and U. Scherf, J. Am. Chem. Soc., 2020, 142, 2404-2412.

93 L. Zhu, D. Zheng, Z. Wang, X. Zheng, P. Fang, J. Zhu, M. Yu, Y. Tong and X. Lu, Adv. Mater., 2018, 30, e1805268.

94 Y. Jiang, Y. P. Deng, R. Liang, J. Fu, D. Luo, G. Liu, J. Li, Z. Zhang, Y. Hu and Z. Chen, Adv. Energy Mater., 2019, 9, 1900911.

95 Z. Pei, Y. Huang, Z. Tang, L. Ma, Z. Liu, Q. Xue, Z. Wang, H. Li, Y. Chen and C. Zhi, Energy Storage Mater., 2019, 20, 234-242.

96 Q. Lu, J. Yu, X. Zou, K. Liao, P. Tan, W. Zhou, M. Ni and Z. Shao, Adv. Funct. Mater., 2019, 29, 1904481.

97 H.-F. Wang, C. Tang, B. Wang, B.-Q. Li, X. Cui and Q. Zhang, Energy Storage Mater., 2018, 15, 124-130.
98 K. Kordek, L. Jiang, K. Fan, Z. Zhu, L. Xu, M. Al-Mamun, Y. Dou, S. Chen, P. Liu, H. Yin, P. Rutkowski and H. Zhao, Adv. Energy Mater., 2019, 9, 1802936.

99 L. Liu, Y. Wang, F. Yan, C. Zhu, B. Geng, Y. Chen and S. 1. Chou, Small Methods, 2019, 4, 1900571.

100 C. Wang, N.-H. Xie, Y. Zhang, Z. Huang, K. Xia, H. Wang, S. Guo, B.-Q. Xu and Y. Zhang, Chem. Mater., 2019, 31, 1023-1029.

101 L. Liu, X. Zhang, F. Yan, B. Geng, C. Zhu and Y. Chen, J. Mater. Chem. A, 2020, 8, 18162-18172.

102 S. Chen, X. Shu, H. Wang and J. Zhang, J. Mater. Chem. A, 2019, 7, 19719-19727.

103 D. Ji, L. Fan, L. Li, N. Mao, X. Qin, S. Peng and S. Ramakrishna, Carbon, 2019, 142, 379-387.

104 K. N. Dinh, Z. Pei, Z. Yuan, V. C. Hoang, L. Wei, Q. Huang, X. Liao, C. Liu, Y. Chen and Q. Yan, J. Mater. Chem. A, 2020, 8, 7297-7308.

105 Z. Yang, C. Zhao, Y. Qu, H. Zhou, F. Zhou, J. Wang, Y. Wu and Y. Li, Adv. Mater., 2019, 31, e1808043.

106 Z.-Y. Yang, Y.-X. Zhang, L. Jing, Y.-F. Zhao, Y.-M. Yan and K.-N. Sun, J. Mater. Chem. A, 2014, 2, 2623.

107 L. Yu, Q. Yi, G. Li, Y. Chen and X. Yang, J. Electrochem. Soc., 2018, 165, A2502-A2509.

108 L. An, Z. Zhang, J. Feng, F. Lv, Y. Li, R. Wang, M. Lu, R. B. Gupta, P. Xi and S. Zhang, J. Am. Chem. Soc., 2018, 140, 17624-17631.

109 Z. Pei, Z. Yuan, C. Wang, S. Zhao, J. Fei, W. Li, J. Chen, C. Wang, R. Qi, Z. Liu and Y. Chen, Angew. Chem., Int. Ed., 2020, 132, 4823-4829.

110 D. Du, S. Zhao, Z. Zhu, F. Li and J. Chen, Angew. Chem., Int. Ed., 2020, 59, 1-6. 